\title{
Synthesis and properties of $\left(2^{\prime} S\right)$ - and $\left(2^{\prime} R\right)-2^{\prime}$-deoxy-2'-C-methyl oligonucleotides
}

\author{
Daniel O. Cicero, ${ }^{\mathrm{a}}$ Mariana Gallo, ${ }^{\mathrm{b}}$ Philippe J. Neuner ${ }^{\mathrm{a}}$ and Adolfo M. Iribarren ${ }^{\mathrm{b}, \mathrm{c}, *}$ \\ ${ }^{a}$ Istituto di Ricerche di Biologia Molecolare P. Angeletti, Via Pontina Km. 30,600, 00040-Pomezia, Rome, Italy \\ ${ }^{\mathrm{b}}$ Instituto de Investigaciones en Ingeniería Genética y Biología Molecular, Vuelta de Obligado 24902 P, 1428 Buenos Aires, Argentina \\ ${ }^{\mathrm{c}}$ Universidad Nacional de Quilmes, Roque Sáenz Peña 180, Bernal, 1876 Pcia. de Buenos Aires, Argentina
}

Received 24 May 2001; accepted 20 July 2001

\begin{abstract}
The synthesis of $\left(2^{\prime} S\right)$ - and $\left(2^{\prime} R\right)-2^{\prime}$-deoxy- $2^{\prime}-C$-methyl- $N^{3}$-(4-t-butylbenzoyl) uridine, $\left(2^{\prime} S\right)-2^{\prime}$-deoxy- $2^{\prime}-C$-methyluridine and $\left(2^{\prime} S\right)-2^{\prime}$-deoxy- $2^{\prime}-C$-methyl- $N^{4}$-isobutyryl cytidine building blocks are here described. The preparation of oligonucleotides carrying these monomers in all positions but $3^{\prime}$-end is presented and the binding affinity between these new fragments and the complementary DNA and RNA sequences is also assessed. $\left(2^{\prime} R\right)$ substituted oligonucleotides did not hybridize with either the complementary DNA or RNA sequences. However, the first derivative of melting curves of hybrids containing $\left(2^{\prime} S\right)$ modified oligonucleotides indicated melting transitions. (C) 2001 Elsevier Science Ltd. All rights reserved.
\end{abstract}

\section{Introduction}

The synthesis of modified oligonucleotides has received a great deal of attention during the last years, due to the very important applications that this class of compounds has displayed as antisense fragments. Several modifications have been proposed in order to obtain molecules resistant to the action of nucleases and that can recognize complementary sequences. ${ }^{1}$ Oligonucleotides based on a nuclease resistant phosphorothioate backbone have proved to be very potent and selective inhibitors of protein expression in vitro as well as in vivo. ${ }^{2,3}$ Despite this therapeutic potential, the detailed analysis of their whole chemical, biochemical and biophysical characteristics still indicates that this class of compounds may not represent the final solution to the problem of designing functional antisense inhibitors. ${ }^{4}$ $2^{\prime}$-Modifications have also proved to have interesting hybridization properties. ${ }^{5} 2^{\prime}-O$-alkyloligoribonucleotides 6,7 which have shown to be resistant to nucleases and to form stable hybrids are widely used both alone and in combination with modified phosphate backbones. ${ }^{8,9}$ The improved hybridization of $2^{\prime}-F$ - and $2^{\prime}-O$-alkyloligonucleotides to complementary RNA has been attributed to the tendency of these electronegative substituents to shift the conformational equilibrium in the sugar moiety towards $\mathrm{C} 3{ }^{\prime}$-endo

Keywords: antisense; modified oligonucleotides; $2^{\prime}$-deoxy-2'-C-alkylnucleotides; hybridization properties.

* Corresponding author. Address: Instituto de Investigaciones en Ingeniería Genética y Biología Molecular, Vuelta de Obligado 2490 2P, 1428 Buenos Aires, Argentina. Tel.: +5411-4783-2871; fax: +5411-47868578; e-mail: airi@dna.uba.ar pucker consistent with the A-form geometry of RNA duplexes. Therefore, the sugar ring puckering seems to constitute an important structural variable to take into account in the design of antisense oligonucleotides. $5,10,11$ We have previously determined the influence of $2^{\prime}-C$ alkyl groups on the sugar conformation of nucleosides in solution based on NMR analysis. ${ }^{12}$ The results showed that the preferred sugar conformation in the case of $\left(2^{\prime} S\right)-2^{\prime}$ deoxy- $2^{\prime}-C$-alkylnucleosides is $\mathrm{C}-3^{\prime}$ endo while the $\left(2^{\prime} R\right)$ isomers exist predominantly in the $\mathrm{C}-2^{\prime}$ endo conformation. These findings could be explained since $2^{\prime}-C$ alkyl substituents tend to attain a pseudoequatorial location for steric reasons while an oxygenated subtituent on $\mathrm{C}-2^{\prime}$ tends to occupy a pseudoaxial site because of the stabilizing O-4'/ $\mathrm{O}-2^{\prime}$ gauche effect. ${ }^{13}$

As far as we know, there is only one report regarding to the synthesis and properties of chimeric sequences containing $2^{\prime}$-deoxy and $2^{\prime}-C$-alkyldeoxynucleotides which exhibited a reduced binding affinity for complementary DNA and RNA. ${ }^{14}$ Despite these results, $\left(2^{\prime} R\right)-2^{\prime}-C$-alkylnucleotides have proved to be useful in the design of modified hammerhead ribozymes. ${ }^{15,16}$ In order to further investigate the properties of oligonucleotides carrying these modifications we carried out preliminary attempts to explore the influence of the $2^{\prime}$-methyl group on the orientation of the base as well on vicinal nucleotides. In view of the results afforded by these preliminary studies, and since differences between the hybridization affinity of oligodeoxynucleotides carrying $2^{\prime}$-modified moieties in certain positions and completely modified sequences has been formerly observed ${ }^{10}$, we consider interesting to further investigate the synthesis and hybridization properties of oligonucleotides carrying 


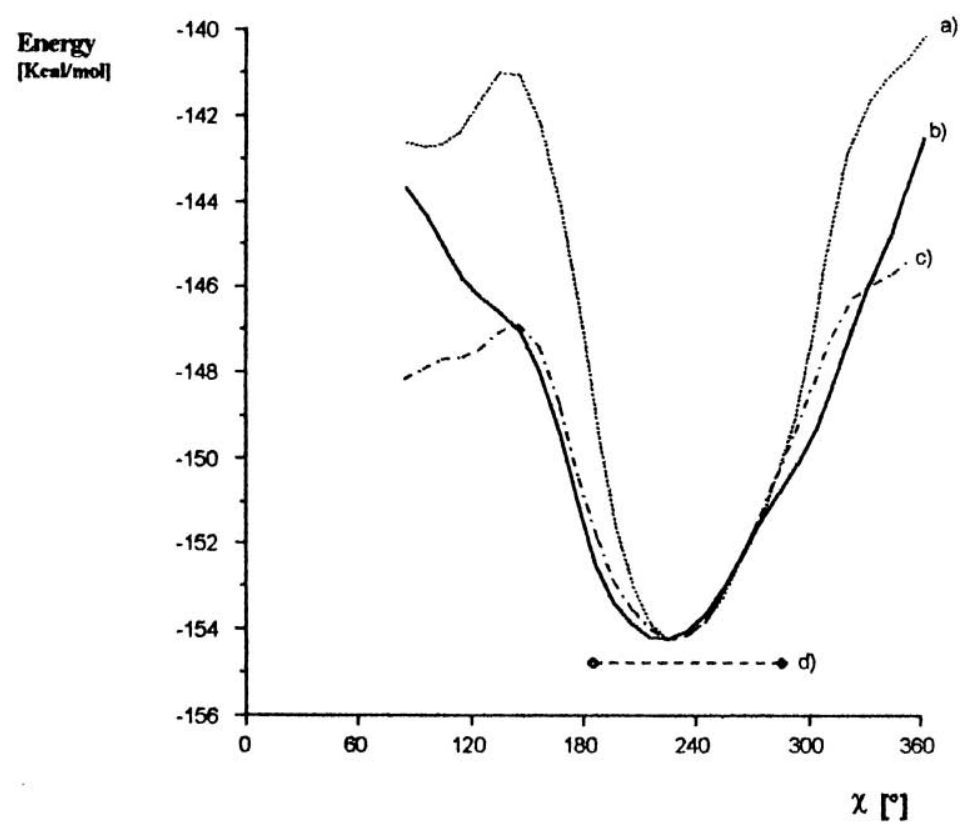

Figure 1. Potential energy as a function of the glycosidic angle $c$ for: (a) $\mathrm{U}_{\left(2^{\prime} S\right) \mathrm{Me}}$, (b) dU and (c) rU. (d) Represents the range of $c$ usually found in natural DNA duplexes.

$2^{\prime}-C$-methyl substituents with both arabino and ribo configurations in all but $3^{\prime}$-positions.

\section{Results and discussion}

Preliminary studies were done in order to explore the effect of the methyl group on the orientation of the base which could produce structural distortions in the case of the $\left(2^{\prime} S\right)$ isomer. The energy of different rotamers around the glycosidic bond was analyzed by means of molecular mechanics calculations. Curves of Fig. 1 show the calculated energy as a function of the glycosidic angle $c$, using a grid step of $10^{\circ}$; the curves for natural and $2^{\prime}-C$-alkyl-modified uridine nucleosides present similar shapes in the range $180-280^{\circ}$. These results suggest that the introduction of the $\left(2^{\prime} S\right)$ methyl group does not impair the orientation of the base within the range of glycosidic angles found in natural duplexes. ${ }^{11}$

A further attempt to predict the hybridization properties of this kind of modified oligonucleotides was made by exploring the interaction of the $2^{\prime}$-methyl group with vicinal nucleotides. The pyrimidine-DNA strand from a DNARNA hybrid constructed using the Builder module of the program Insight, ${ }^{17}$ was modified by direct replacement of the $2^{\prime}-\beta$-hydrogen by a methyl group. An analysis of bumping interactions showed that the $2^{\prime}$-methyl group most likely sterically interferes with the $3^{\prime}$-nucleotide in the oligomer, though these minor interactions would not justify an absence of hybridization. On the other hand, the substitution of the $2^{\prime}-\alpha$-hydrogen produced strong destabilizing interactions with vicinal nucleotides. To verify experimentally this preliminary conclusions we decided to synthesize oligonucleotides carrying $\left(2^{\prime} S\right)$ - and $\left(2^{\prime} R\right)-C$-methylnucleotides in all positions but $3^{\prime}$-end.

Fig. 2 shows the synthesis of the uridine building blocks $\mathbf{5 a}$ and $\mathbf{5 b}$ starting from the mixture of $\left(2^{\prime} S\right)$ - and $\left(2^{\prime} R\right)-2^{\prime}-$ deoxy-3',5'-O-(tetraisopropyldisiloxane-1,3-diyl)-2'-Cmethyluridine (1a and $\mathbf{1 b}){ }^{18}$

Reaction of the mixture of $\mathbf{1 a}$ and $\mathbf{1 b}$ with 4-t-butylbenzoyl chloride yielded compounds $\mathbf{2 a}$ and $\mathbf{2} \mathbf{b}$. The introduction of the lipophilic acyl group made chromatographic separation of both diasteromers possible, and also conferred additional protection for the lactam function during the longer coupling times required for $2^{\prime}$-substituted nucleotides. ${ }^{19}$ The disiloxane bridge of the isolated isomers was removed with tetrabutylammonium fluoride to afford compounds $\mathbf{3 a}$ (97\% yield) and $\mathbf{3 b}$ (69\% yield). The configuration at $C-2^{\prime}$ was assigned by comparing the NMR data of $\mathbf{3 a}$ and $\mathbf{3 b}$ with those of $\left(2^{\prime} S\right)$ - and $\left(2^{\prime} R\right)-2^{\prime}-$ deoxy-2'-C-2'-methyluridine ${ }^{18}$ (data not shown).

$5^{\prime}-O$-Dimethoxytrityl derivatives $(\mathbf{4 a}$ and $\mathbf{4 b})$, as well as $3^{\prime}$ $O$-phosphoramidites, $\mathbf{5 a}$ and $\mathbf{5 b}$, were prepared following standard procedures.

In order to assess the hybridization properties of completely modified oligonucleotides carrying $2^{\prime}$-modified building blocks in all but $3^{\prime}$-position the following fragments were synthesized: $\left(\mathrm{U}_{\left(2^{\prime} R\right) M e}\right)_{10} \mathrm{~T}(\mathbf{I})$, and $\left(\mathrm{U}_{\left(2^{\prime} S\right) M e}\right)_{10} \mathrm{~T}(\mathbf{I I})$, (where $\mathrm{U}_{\left(2^{\prime} R\right) \mathrm{Me}}=\left(2^{\prime} R\right)-2^{\prime}-$ deoxy- $2^{\prime}-C$-methyluridine; $\mathrm{U}_{\left(2^{\prime} S\right) \mathrm{Me}}=\left(2^{\prime} S\right)$ $2^{\prime}$-deoxy-2'-C-methyluridine and $\mathrm{T}=$ thymidine). Sugarmodified phosphoramidites $\mathbf{5 a}$ and $\mathbf{5 b}$ were used as building blocks in an automated DNA synthesizer. Due to the steric interference caused by the bulky $2^{\prime}-C$-alkyl substituent, the normal coupling cycle was modified to ensure high coupling yield of the protected monomers (7 min). Deprotection and purification were performed as described in Section 3.

On the other hand, with the goal of analyzing the hybridization behavior of a mixed cytidine-uridine sequence carrying $\left(2^{\prime} S\right)-2^{\prime}$-deoxy-2'-C-methylnucleotides, the synthesis of the cytidine building block was performed (Fig. 3). The 


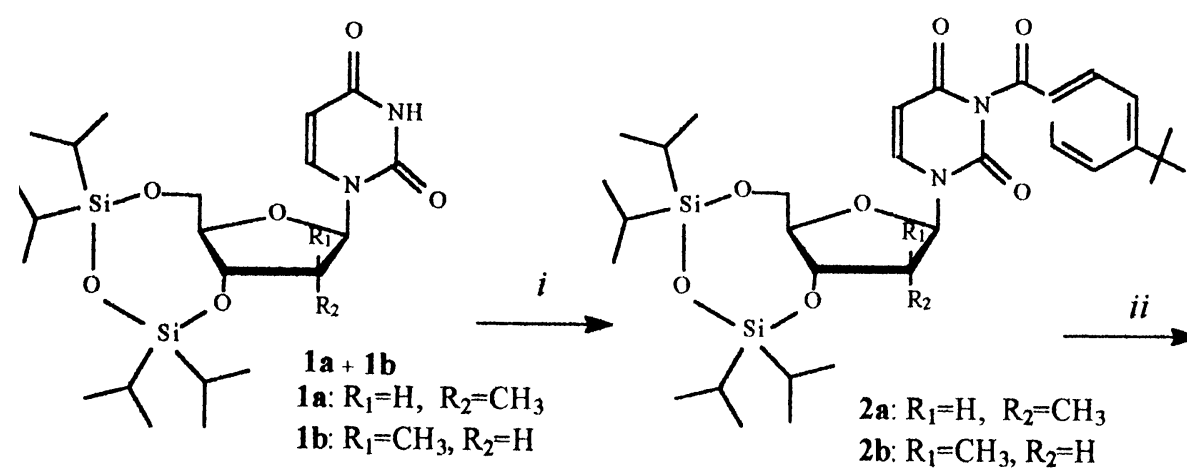

2b: $\mathrm{R}_{1}=\mathrm{CH}_{3}, \mathrm{R}_{2}=\mathrm{H}$

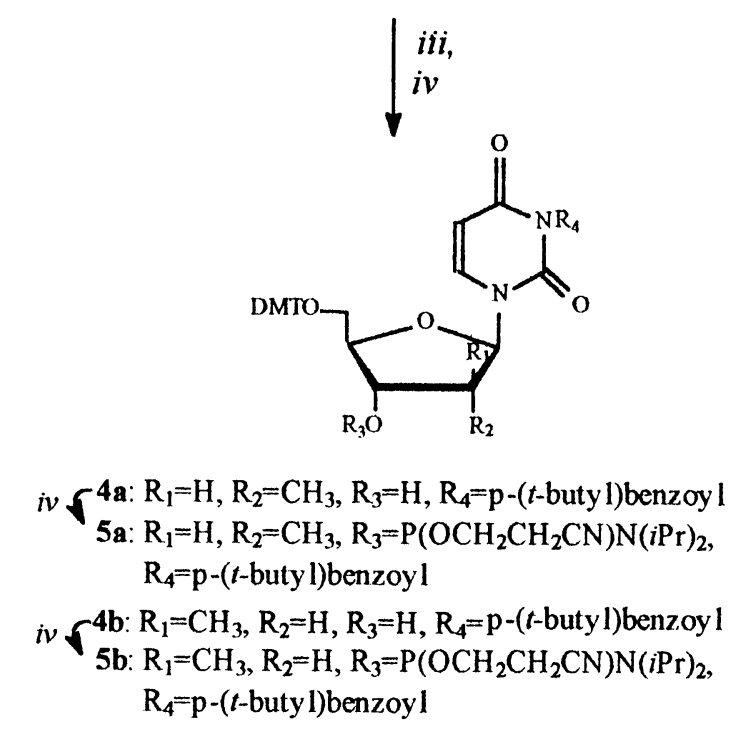

Figure 2. Reaction scheme for the preparation of $\left(2^{\prime} S\right)$ - and $\left(2^{\prime} R\right)-2^{\prime}$-deoxy- $2^{\prime}-C$-methyl- $N^{3}$-(4-t-butylbenzoyl)uridine building blocks. Reagents: (i) tetrabutylammonium bromide, $\mathrm{Na}_{2} \mathrm{CO}_{3}$ and 4- $t$-butylbenzoyl chloride in dichloromethane/ $\mathrm{H}_{2} \mathrm{O}(1: 1$, v/v); (ii) tetrabutylammonium fluoride in tetrahydrofuran; (iii) $4,4^{\prime}$-dimethoxytrityl chloride and triethylamine in pyridine; (iv) 2-cyanoethoxy $N, N$-diisopropylaminochlorophosphine and $N, N$-diisopropylethylamine in 1,2 dichloroethane.

mixture of compounds $\mathbf{1 a}$ and $\mathbf{1 b}$ was treated with 2-nitrophenol and diazabicyclo[2,2,2]octane in 1,2-dichloroethane/triethylamine to afford the two diasteromers of the corresponding $\mathrm{O}^{4}$-nitrophenylnucleosides. This mixture could be resolved by silica gel column chromatography to afford pure compound $\mathbf{6}$ which was the common intermediate for the synthesis of the uridine (9) and cytidine phosphoramidites (12). Using the same conditions described above for the preparation of the 2'-modified homooligomers, the synthesis of (UCCUCCCUCUUCCUCUCC $)_{\left(2^{\prime} S\right) \mathrm{Me}} \mathrm{dC}$ (III) (where $\mathrm{C}_{\left(2^{\prime} S\right) \mathrm{Me}}=\left(2^{\prime} S\right)-2^{\prime}$-deoxy-2'$C$-methylcytidine; $\mathrm{U}_{\left(2^{\prime} S\right) \mathrm{Me}}=\left(2^{\prime} S\right)-2^{\prime}$-deoxy-2'-C -methyluridine and $\mathrm{d} C=$ deoxycytidine) was performed. No difference in the yield of the oligomers between the preparation using lactam protected uridine building block $\mathbf{5 b}$, and the lactam unprotected 9 were observed.

The thermal stability of the hybrids formed between these oligonucleotides and the complementary sequences was tested. Oligonucleotide $\mathbf{I}$ did not hybridize with $\mathrm{dA}_{11}$ or $-\mathrm{rA}_{11}$ sequences. This result is in agreement with that reported by Schmidt et al. for the case of chimeric oligonucloetides carrying $\left(2^{\prime} R\right)-2^{\prime}-C$-alkyloligonucleotide. ${ }^{14} \mathrm{On}$ the other hand, fragment II showed a thermal transition with both $\mathrm{dA}_{11}$ and $\mathrm{rA}_{11}$. In both cases, the observed difference in absorbance is much smaller than those obtained for the hybrids $\mathrm{T}_{11} / \mathrm{dA}_{11}$ and $\mathrm{T}_{11} / \mathrm{rA}_{11}$, but transitions were evidenced by the first derivatives of the corresponding melting curves. These results are not due to particular single strand structures, since the melting curves of the single oligonucleotides did not show any transition.

According to the first derivatives, the melting points measured were the following: $\mathrm{T}_{11} / \mathrm{dA}_{11}: 24.0^{\circ} \mathrm{C} ; \mathbf{I I} / \mathrm{dA}_{11}$ : $25.9^{\circ} \mathrm{C} ; \mathrm{T}_{11} / \mathrm{rA}_{11}: 16.5^{\circ} \mathrm{C} ; \mathbf{I I} / \mathrm{rA}_{11}: 26.2^{\circ} \mathrm{C}$. These results show that only the modified oligonucleotides carrying $\left(2^{\prime} S\right)$-alkyl groups presented good hybridization properties, with melting points higher than those measured for the natural DNA-DNA and DNA-RNA hybrids. It also suggests that although the stacking interactions seem to be disturbed by the presence of the methyl group, the hybrids containing modified uridines are thermally more stable than the corresponding hybrids carrying thymidines.

In order to assess the hybridization behavior of a mixed cytidine-uridine sequence, the hybridization behavior of the mixed sequence III with the complementary RNA olilgonucleotide was performed. In the case of the hybrid formed between oligonucleotide III and the corresponding complementary RNA sequence the melting temperature 


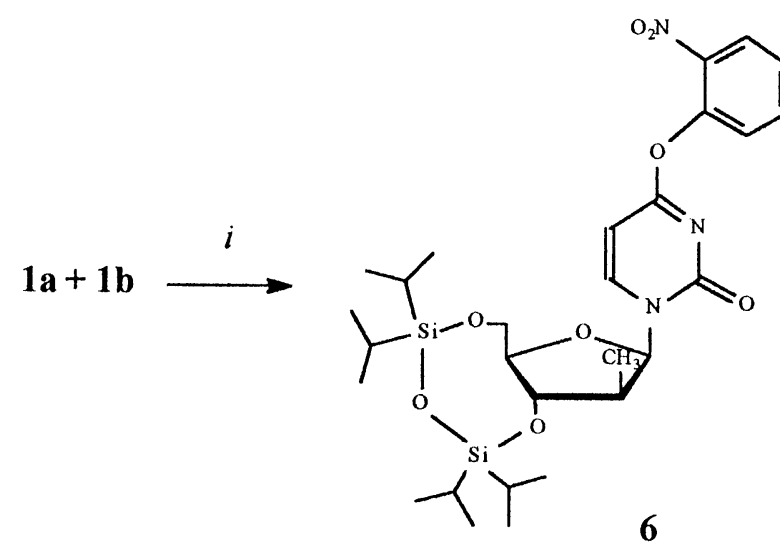

$\left[+\left(2^{\prime} \mathbf{R}\right)\right.$-isomer $]$

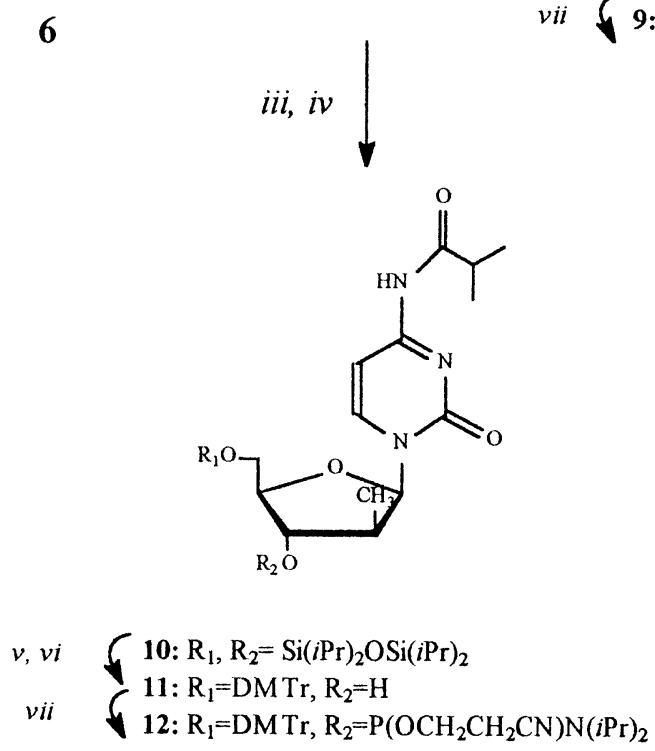

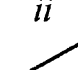

ii
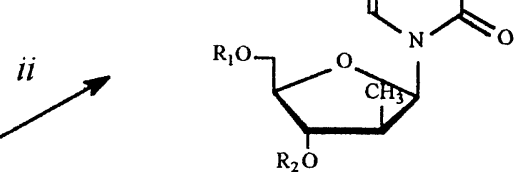

$v, v i \quad \begin{aligned} & \text { 7: } \mathrm{R}_{1}, \mathrm{R}_{2}=\mathrm{Si}(i \mathrm{Pr})_{2} \mathrm{OSi}(i \mathrm{Pr})_{2} \\ & \text { 8: } \mathrm{R}_{1}=\mathrm{DMT}, \mathrm{R}_{2}=\mathrm{H}\end{aligned}$

9: $\mathrm{R}_{1}=\mathrm{DMTr}, \mathrm{R}_{2}=\mathrm{P}\left(\mathrm{OCH}_{2} \mathrm{CH}_{2} \mathrm{CN}\right) \mathrm{N}(\mathrm{iPr}$ 
Oligonucleotides were synthesized on a 380B Applied Biosystems synthesizer using $\beta$-cyanoethyl phosphoramidite chemistry. Commercially available (Millipore) derivatized CPG-linked to either $2^{\prime}$-deoxy or ribonucleosides were used. Coupling efficiency was in all the cases higher than $98 \%$. Deprotection of oligonucleotides was achieved by treatment with concentrated aqueous ammonia at $55^{\circ} \mathrm{C}$, overnight. Oligonucleotides (trityl-on), which showed a major peak, were purified by reverse phase HPLC on a Nova-Pak C-18 column $(8 \times 10$ Radial-Pak Cartridge, $4 \mathrm{~mm}$ ) using a gradient of $16-60 \%$ of acetonitrile in $0.1 \mathrm{M}$ triethylammonium acetate buffer $\mathrm{pH}$ 7. Cleavage of DMTr group was accomplished by treatment with $80 \%$ acetic acid for $30 \mathrm{~min}$. The solution was diluted with an equal volume of water, washed three times with two volumes of diethyl ether, and the aqueous layer was lyophilized.

Melting curves were measured on a Cary 3E (Varian) spectrophotometer with temperature controller. Hybrids were previously annealed by heating the solution of the oligonucleotides $3 \mathrm{mM}$ of each strand in $100 \mathrm{mM} \mathrm{NaCl}$, $10 \mathrm{mM}$ Tris buffer $(\mathrm{pH}=7.1)$ at $80^{\circ} \mathrm{C}$ for $2 \mathrm{~min}$ and subsequent cooling for $40 \mathrm{~min}$ to a final temperature of $5^{\circ} \mathrm{C}$. The melting curves were obtained by measuring the absorbance at $260 \mathrm{~nm}$, using a heating rate of $0.5^{\circ} \mathrm{C} / \mathrm{min}$. Melting temperatures $\left(T_{\mathrm{m}}\right)$ were determined as the maximum of the first derivative of the plot of absorbance vs. temperature.

Microanalysis of previously recrystallized samples was done using an automatic analyzer (Fisons Instruments). Molecular mechanics calculations were performed using the program Discover (Biosym). The energy minimizations were performed in vacuo, using the AMBER force field. Curves of Fig. 1 were obtained using a grid step of $10^{\circ}$. At each value of the glycosidic angle, the structure was minimized to an rms energy gradient of $0.001 \mathrm{kcal} / \mathrm{mol} \mathrm{A}$, using a $10 \mathrm{kcal} / \mathrm{mol}$ harmonic restraint to maintain the appropriate value of $c$.

3.1.1. $3^{\prime}, 5^{\prime}-O$-(Tetraisopropyldisiloxane-1,3-diyl)-(2'R)and $\quad-\left(2^{\prime} S\right)-2^{\prime}-$ deoxy-2 $-C$-methyl- $N^{3}-(4-t$-butylbenzoyl $)$ uridine (2a and $2 \mathbf{b})$. To a vigorously stirred mixture of $3^{\prime}, 5^{\prime}-O$-(tetraisopropyldisiloxane-1,3-diyl)- $\left(2^{\prime} R\right)$ - and $\left(2^{\prime} S\right)$ $2^{\prime}$-deoxy-2'-C-methyluridine (1a, 1b) $(30 \mathrm{mg}, 0.06 \mathrm{mmol})$, tetrabutylammonium bromide $(2 \mathrm{mg}), \mathrm{Na}_{2} \mathrm{CO}_{3}(50 \mathrm{mg}$, $0.47 \mathrm{mmol})$ and dichloromethane $/ \mathrm{H}_{2} \mathrm{O}(2 \mathrm{~mL}, 1: 1, \mathrm{v} / \mathrm{v})$, was added dropwise 4-t-butylbenzoyl chloride $(20 \mathrm{~mL}$, $0.10 \mathrm{mmol}$ ). The reaction mixture was stirred overnight at room temperature. The organic phase was separated, washed with $\mathrm{H}_{2} \mathrm{O}(5 \mathrm{~mL})$ and left with stirring overnight (in order to obtain the thermodynamically more stable $N^{3}$-acyl derivative ${ }^{21}$ ). The solvent was evaporated in vacuo and the residue chromatographied on silica gel column using as eluant a gradient of petroleum ether/ethyl acetate $9: 1$ to $7: 1(\mathrm{v} / \mathrm{v})$. Pure compounds $2 \mathbf{a}(12 \mathrm{mg}$, $0.018 \mathrm{mmol})$ and $\mathbf{2 b}(19 \mathrm{mg}, 0.029 \mathrm{mmol})$ were obtained. ${ }^{13} \mathrm{C}$ NMR spectrum of $\mathbf{2 a}\left(\mathrm{CDCl}_{3}\right):(\delta, \mathrm{ppm}) 168.8$ ( $t$-butylbenzoyl); 162.0 (C-4); 154.6 ( $t$-butylbenzoyl); 149.2 (C-2); 138.8 (C-6); 131.7, 128.8, 124.4 ( $t$-butylbenzoyl); 102.1

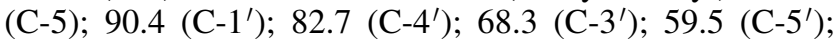
$43.4\left(\mathrm{C}-2^{\prime}\right) ; 34.8$ and 31.4 ( $t$-butylbenzoyl); 17.3-12.3 (isopropyls), 10.5 (2'-methyl). ${ }^{1} \mathrm{H}$ NMR spectrum of $2 \mathbf{a}$ $\left(\mathrm{CDCl}_{3}\right):(\delta, \mathrm{ppm}) 0.81-1.23\left(\mathrm{~m}, 28 \mathrm{H},-\mathrm{CHs},-\mathrm{CH}_{3} \mathrm{~s}\right.$, disiloxane); $0.87\left(\mathrm{~d}, 3 \mathrm{H},-\mathrm{CH}_{3}, J_{\mathrm{CH} 3,2^{\prime}}=7 \mathrm{~Hz}\right) ; 1.32(\mathrm{~s}$, $9 \mathrm{H},-\mathrm{CH}_{3} \mathrm{~s}, t$-butyl); $2.23\left(\mathrm{~m}, 1 \mathrm{H}, \mathrm{H}-2^{\prime}\right) ; 3.87$ (dd, $1 \mathrm{H}, \mathrm{H}-$ $\left.5^{\prime}, \quad J_{5^{\prime}, 5^{\prime \prime}}=13 \mathrm{~Hz} ; \quad J_{4^{\prime}, 5^{\prime}}=1 \mathrm{~Hz}\right) ; 4.00 \quad\left(\mathrm{dd}, 1 \mathrm{H}, \mathrm{H}-5^{\prime \prime}\right.$, $\left.J_{5^{\prime}, 5^{\prime \prime}}=13 \mathrm{~Hz} ; J_{4^{\prime}, 5^{\prime \prime}}=1 \mathrm{~Hz}\right) ; 4.15\left(\mathrm{~m}, 1 \mathrm{H}, \mathrm{H}-4^{\prime}\right) ; 4.42(\mathrm{~m}$, $\left.1 \mathrm{H}, \mathrm{H}-3^{\prime}\right) ; 5.72\left(\mathrm{~d}, 1 \mathrm{H}, \mathrm{H}-5, J_{6,5}=8 \mathrm{~Hz}\right) ; 5.95(\mathrm{~d}, 1 \mathrm{H}, \mathrm{H}-$ $\left.1^{\prime}, J_{1,2}=9 \mathrm{~Hz}\right) ; 7.40$ (d, 2H, $t$-butylbenzoyl, $\left.J=9 \mathrm{~Hz}\right) ; 7.81$ (d, $\left.1 \mathrm{H}, \mathrm{H}-6, J_{6,5}=8 \mathrm{~Hz}\right) ; 8.02$ (d, 2H, $t$-butylbenzoyl, $J=9 \mathrm{~Hz}) .{ }^{13} \mathrm{C}$ NMR spectrum of $\mathbf{2 b}\left(\mathrm{CDCl}_{3}\right)$ : $(\delta, \mathrm{ppm})$ 168. 9 (t-butylbenzoyl); 161.6 (C-4); 154.8 ( $t$-butylbenzoyl); 149.0 (C-2); 138.5 (C-6); 131.7, 128.8 and 124.2 ( $t$-butylbenzoyl); $102.4(\mathrm{C}-5) ; 85.8,84.3\left(\mathrm{C}-1^{\prime}\right.$ and $\left.\mathrm{C}-4^{\prime}\right) ; 72.1\left(\mathrm{C}-3^{\prime}\right) ; 60.3\left(\mathrm{C}-5^{\prime}\right) ; 44.1\left(\mathrm{C}-2^{\prime}\right) ; 34.9,31.5$ ( $t$-butylbenzoyl); 17.4-12.3 (isopropyls), 11.1 (2'-methyl). ${ }^{1} \mathrm{H}$ NMR spectrum of $\mathbf{2 b}\left(\mathrm{CDCl}_{3}\right):(\delta, \mathrm{ppm}) 0.80-1.22(\mathrm{~m}$, $28 \mathrm{H},-\mathrm{CHs},-\mathrm{CH}_{3} \mathrm{~s}$, disiloxane); $0.92\left(\mathrm{~d}, 3 \mathrm{H},-\mathrm{CH}_{3}\right.$, $\left.J_{\mathrm{CH} 3,2^{\prime}}=7 \mathrm{~Hz}\right) ; 1.34\left(\mathrm{~s}, 9 \mathrm{H},-\mathrm{CH}_{3} \mathrm{~s}, t\right.$-butyl $) ; 2.73(\mathrm{~m}, 1 \mathrm{H}$, $\left.\mathrm{H}-2^{\prime}\right) ; 3.75\left(\mathrm{dd}, 1 \mathrm{H}, \mathrm{H}-5^{\prime}, J_{5^{\prime}, 5^{\prime \prime}}=12 \mathrm{~Hz} ; J_{4^{\prime}, 5^{\prime}}=1 \mathrm{~Hz}\right) ; 3.96$ $\left(\mathrm{dd}, 1 \mathrm{H}, \mathrm{H}-5^{\prime \prime}, J_{5^{\prime}, 5^{\prime \prime}}=13 \mathrm{~Hz} ; J_{4^{\prime}, 5^{\prime \prime}}=1 \mathrm{~Hz}\right) ; 4.01(\mathrm{~m}, 1 \mathrm{H}$, $\left.\mathrm{H}-4^{\prime}\right) ; 4.22\left(\mathrm{~m}, 1 \mathrm{H}, \mathrm{H}-3^{\prime}\right) ; 5.81\left(\mathrm{~d}, 1 \mathrm{H}, \mathrm{H}-5, J_{6,5}=8 \mathrm{~Hz}\right)$; $6.25\left(\mathrm{~d}, 1 \mathrm{H}, \mathrm{H}-1^{\prime}, J_{1,2}=8 \mathrm{~Hz}\right) ; 7.38$ (d, 2H, $t$-butylbenzoyl, $J=9 \mathrm{~Hz}) ; 7.76$ (d, 1H, H-6, $\left.J_{6,5}=8 \mathrm{~Hz}\right) ; 8.11$ (d, 2H, $t$-butylbenzoyl, $J=9 \mathrm{~Hz}$ ). Anal. calcd for $\mathrm{C}_{33} \mathrm{H}_{52} \mathrm{O}_{7} \mathrm{~N}_{2} \mathrm{Si}_{2}(\mathbf{2 a}$ and 2b): C, 61.46; H, 8.13; N, 4.34. Found for 2a: C, 61.50; H, 8.15; N, 4.40; 2b: C, 61.50; H, 8.10; N, 4.45 .

3.1.2. $\left(2^{\prime} R\right)-2^{\prime}$-Deoxy-2' $C$-methyl- $N^{3}$-(4-t-butylbenzoyl) uridine (3a). $3^{\prime}, 5^{\prime}-O$-(Tetraisopropyldisiloxane-1,3-diyl)$\left(2^{\prime} R\right)-2^{\prime}$-deoxy-2'-C-methyl- $N^{3}$-(4-t-butylbenzoyl) uridine (2a) $(300 \mathrm{mg}, 0.46 \mathrm{mmol})$ was dissolved in dry tetrahydrofuran $(1.4 \mathrm{~mL})$ and tetrabutylammonium fluoride $(1 \mathrm{mmol})$ was added with stirring and exclusion of moisture. After $5 \mathrm{~min}$ the reaction mixture was quenched with $\mathrm{MeOH} /$ pyridine $/ \mathrm{H}_{2} \mathrm{O}(2.8 \mathrm{~mL}, 3: 3: 1 \mathrm{v} / \mathrm{v} / \mathrm{v})$, and stirred for $5 \mathrm{~min}$. Pyridinium form Dowex 50×2-200 resin (15 g) was added to the solution and the mixture was stirred for $15 \mathrm{~min}$. The resin was filtered off and washed with $\mathrm{MeOH}(2 \times 2.8 \mathrm{~mL})$. Combined filtrate and washings were evaporated in vacuo and the crude product was purified by silica gel column, eluting with a gradient of $\mathrm{MeOH}$ from 2 to $5 \%$ in dichloromethane. Pure title compound was obtained (180 mg) in $97 \%$ yield. ${ }^{13} \mathrm{C}$ NMR spectrum of $\mathbf{3 a}\left(\mathrm{CDCl}_{3}\right):(\delta, \mathrm{ppm})$ 168.8 ( $t$-butylbenzoyl); 162.0 (C-4); 154.7 ( $t$-butylbenzoyl); 148.9 (C-2); 139.0 (C-6); 131.7, 128.8, 124.4 ( $t$-butylbenzoyl); 102.4 (C-5); 89.2, $87.8\left(\mathrm{C}-1^{\prime}\right.$ and $\left.\mathrm{C}-4^{\prime}\right) ; 72.9$ $\left(\mathrm{C}-3^{\prime}\right) ; 61.0\left(\mathrm{C}-5^{\prime}\right) ; 43.9\left(\mathrm{C}-2^{\prime}\right)$; 35.0, 31.5 ( $t$-butylbenzoyl), 10.3 (2'-methyl). ${ }^{1} \mathrm{H}$ NMR spectrum of 3a $\left(\mathrm{CDCl}_{3}\right):(\delta$, ppm) $0.91\left(\mathrm{~d}, 3 \mathrm{H}, \mathrm{CH}_{3}, J_{\mathrm{CH} 3,2^{\prime}}=7 \mathrm{~Hz}\right) ; 1.32(\mathrm{~s}, 9 \mathrm{H}$, $-\mathrm{CH}_{3} \mathrm{~s}, t$-butyl); $2.21\left(\mathrm{~m}, 1 \mathrm{H}, \mathrm{H}-2^{\prime}\right) ; 3.70\left(\mathrm{~m}, 2 \mathrm{H}, \mathrm{H}-5^{\prime}\right.$ and $\mathrm{H}-5^{\prime \prime}$ ); 3.83 (ddd, $1 \mathrm{H}, \mathrm{H}-4^{\prime}, J_{5^{\prime \prime}-4^{\prime}}=J_{5^{\prime}-4^{\prime}}=4 \mathrm{~Hz}, J_{3^{\prime}-4^{\prime}}=2 \mathrm{~Hz}$ ); $4.04\left(\mathrm{dd}, 1 \mathrm{H}, \mathrm{H}-3^{\prime}, J_{2^{\prime}, 3^{\prime}}=6 \mathrm{~Hz}, J_{3^{\prime}-4^{\prime}}=2 \mathrm{~Hz}\right) ; 5.02$ (brs, $1 \mathrm{H}$, $-\mathrm{OH}) ; 5.21$ (brs, $1 \mathrm{H},-\mathrm{OH}) ; 5.65$ (d, $1 \mathrm{H}, \mathrm{H}-5, J_{6,5}=8 \mathrm{~Hz}$ ); $5.75\left(\mathrm{~d}, 1 \mathrm{H}, \mathrm{H}-1^{\prime}, J_{1,2}=9 \mathrm{~Hz}\right) ; 7.39$ (d, 2H, $t$-butylbenzoyl, $J=9 \mathrm{~Hz}) ; 7.83$ (d, 1H, H-6, $\left.J_{6,5}=8 \mathrm{~Hz}\right) ; 8.05$ (d, 2H, $t$-butylbenzoyl, $J=9 \mathrm{~Hz}$ ).

3.1.3. (2'S)-2'-Deoxy-2'-C-methyl- $N^{3}$-(4-t-butylbenzoyl) uridine (3b). $3^{\prime}, 5^{\prime}-O$-(Tetraisopropyldisiloxane-1,3-diyl)$\left(2^{\prime} S\right)-2^{\prime}$-deoxy-2'-C-methyl- $N^{3}$-(4-t-butylbenzoyl)uridine (2b) $(510 \mathrm{mg}, 0.79 \mathrm{mmol})$ was desilylated and purified according to the procedure used to prepare compound 3a above to yield pure title compound $(220 \mathrm{mg}, 69 \%) .{ }^{13} \mathrm{C}$ 
NMR spectrum of $\mathbf{3 b}\left(\mathrm{CDCl}_{3}\right)$ : $(\delta$, ppm) 168.9 ( $t$-butylbenzoyl); 161.5 (C-4); 154.9 ( $t$-butylbenzoyl); 148.9 (C-2); 139.3 (C-6); 131.7, 128.5, 124.3 ( $t$-butylbenzoyl); 102.6 (C-5); $86.0\left(\mathrm{C}-1^{\prime}\right) ; 83.7\left(\mathrm{C}-4^{\prime}\right) ; 73.5\left(\mathrm{C}-3^{\prime}\right) ; 61.9$ $\left(\mathrm{C}-5^{\prime}\right) ; 45.1\left(\mathrm{C}-2^{\prime}\right) ; 34.8,31.3$ (t-butylbenzoyl), 11.1 (2'-methyl). ${ }^{1} \mathrm{H}$ NMR spectrum of $\mathbf{3 b}\left(\mathrm{CDCl}_{3}\right):(\delta, \mathrm{ppm})$ $0.85\left(\mathrm{~d}, 3 \mathrm{H}, \mathrm{CH}_{3}, J_{\mathrm{CH} 32^{\prime}}=7 \mathrm{~Hz}\right) ; 1.34\left(\mathrm{~s}, 9 \mathrm{H},-\mathrm{CH}_{3} \mathrm{~s}\right.$, $t$-butyl); $2.48\left(\mathrm{~m}, 1 \mathrm{H}, \mathrm{H}-2^{\prime}\right) ; 3.65\left(\mathrm{~m}, 2 \mathrm{H}, \mathrm{H}-5^{\prime}\right.$ and $\left.\mathrm{H}-5^{\prime \prime}\right)$; $3.75\left(\mathrm{~m}, 1 \mathrm{H}, \mathrm{H}-4^{\prime}\right) ; 3.80\left(\mathrm{~m}, 1 \mathrm{H}, \mathrm{H}-3^{\prime}\right) ; 5.14$ (brs, $1 \mathrm{H}$, $-\mathrm{OH}) ; 5.35$ (brs, $1 \mathrm{H},-\mathrm{OH}) ; 5.63\left(\mathrm{~d}, 1 \mathrm{H}, \mathrm{H}-5, J_{6.5}=8 \mathrm{~Hz}\right)$; $6.18\left(\mathrm{~d}, 1 \mathrm{H}, \mathrm{H}-1^{\prime}, J_{1.2}=8 \mathrm{~Hz}\right) ; 7.41$ (d, $2 \mathrm{H}, t$-butylbenzoyl, $J=9 \mathrm{~Hz}) ; 8.00$ (d, $\left.1 \mathrm{H}, \mathrm{H}-6, J_{6,5}=8 \mathrm{~Hz}\right) ; 8.15$ (d, $2 \mathrm{H}, t$-butylbenzoyl, $J=9 \mathrm{~Hz})$. Anal. calcd for $\mathrm{C}_{21} \mathrm{H}_{26} \mathrm{O}_{6} \mathrm{~N}_{2}(\mathbf{3 a}$ and $\mathbf{3 b})$ : C, 62.67; H, 6.51; N, 6.96. Found for 3a: C, 62.71; H, 6.38; N, 7.01; 3b: C, 62.75; H, 6.44; N, 6.94.

3.1.4. 5'-O-Dimethoxytrityl-(2'R)-2' -deoxy-2' $C$-methyl$N^{3}$-(4-t-butylbenzoyl)uridine (4a). $\left(2^{\prime} R\right)-2^{\prime}$-Deoxy- $2^{\prime}-C$ methyl- $N^{3}$-(4-t-butylbenzoyl) uridine $3 \mathbf{a}$ ( $\left.2 \mathrm{~g}, 5 \mathrm{mmol}\right)$ was dried by evaporation of pyridine in vacuo. Anhydrous pyridine $(20 \mathrm{~mL})$, triethylamine $(10 \mathrm{mmol})$ and $4,4^{\prime}-$ dimethoxytrityl chloride $(6 \mathrm{mmol})$ were added with stirring and exclusion of moisture. The reaction was checked by TLC and after completion quenched by addition of $\mathrm{MeOH}$ and evaporated to dryness. The residue was dissolved in $(50 \mathrm{~mL})$ and washed with $1 \mathrm{M}$ aqueous $\mathrm{NaHCO}_{3}$ $(2 \times 50 \mathrm{~mL})$. The organic layer was dried $\left(\mathrm{Na}_{2} \mathrm{SO}_{4}\right)$, filtered and solvent was removed in vacuo. The residue was purified by column cromatography on silica gel using $50 \%$ ethyl acetate in petroleum ether containing $1 \%$ triethylamine as eluant $(63 \%) .{ }^{13} \mathrm{C}$ NMR spectrum of $\mathbf{4 a}\left(\mathrm{CDCl}_{3}\right):(\delta, \mathrm{ppm})$ 168.9 ( $t$-butylbenzoyl); $161.8(\mathrm{C}-4) ; 158.5\left(\mathrm{C}_{\mathrm{Ar}}-\mathrm{OCH}_{3}\right.$, DMTr); 154.7 ( $t$-butylbenzoyl); 149.3 (C-2); 144.2 (Ar, DMTr); 140.2 (C-6); 135.3, 130.0, 128.6, 128.0, 127.3 (Ar, DMTr + butylbenzoyl); 131.7, 124.4 ( $t$-butylbenzoyl); 113.1 (Ar, DMTr); 102.3 (C-5); 86.6 (C-Ar 3 , DMTr); 89.3 $\left(\mathrm{C}-1^{\prime}\right) ; 82.4\left(\mathrm{C}-4^{\prime}\right) ; 70.0\left(\mathrm{C}-3^{\prime}\right) ; 60.2\left(\mathrm{C}-5^{\prime}\right) ; 55.1\left(-\mathrm{OCH}_{3}\right.$, DMTr); 44.5 (C-2'); 34.7, 31.3 ( $t$-butylbenzoyl), 10.4 (2'methyl). ${ }^{1} \mathrm{H}$ NMR spectrum of $4 \mathbf{a}\left(\mathrm{CDCl}_{3}\right):(\delta, \mathrm{ppm}) 0.95$ $\left(\mathrm{d}, 3 \mathrm{H},-\mathrm{CH}_{3}, J_{\mathrm{CH} 32^{\prime}}=7 \mathrm{~Hz}\right) ; 1.30\left(\mathrm{~s}, 9 \mathrm{H},-\mathrm{CH}_{3} \mathrm{~s}, t\right.$-butylbenzoyl); 2.45 (brs, $\left.1 \mathrm{H}, \mathrm{OH}-3^{\prime}\right) ; 2.20$ (m, 1H, H-2'); 3.25 (m, 1H, H-5'); $3.45\left(\mathrm{~m}, 1 \mathrm{H}, \mathrm{H}-5^{\prime \prime}\right) ; 3.81\left(\mathrm{~s}, 6 \mathrm{H},-\mathrm{OCH}_{3}\right)$; $4.00\left(\mathrm{~m}, 1 \mathrm{H}, \mathrm{H}-4^{\prime}\right) ; 4.31\left(\mathrm{~m}, 1 \mathrm{H}, \mathrm{H}-3^{\prime}\right) ; 5.45(\mathrm{~d}, 1 \mathrm{H}, \mathrm{H}-5$, $\left.J_{5,6}=8 \mathrm{~Hz}\right) ; 6.15\left(\mathrm{~d}, 1 \mathrm{H}, \mathrm{H}-1^{\prime}, J_{1^{\prime}, 2^{\prime}}=8 \mathrm{~Hz}\right) ; 6.80(\mathrm{~d}, 4 \mathrm{H}, \mathrm{Ar}-$ $\left.\mathrm{OCH}_{3}, \mathrm{DMT}, J=9 \mathrm{~Hz}\right) ; 7.21-7.35\left(\mathrm{~m}, 11 \mathrm{H}, \mathrm{Ar}+\mathrm{Ar}-\mathrm{OCH}_{3}\right.$, DMT and Ar, $t$-butylbenzoyl); $8.03\left(\mathrm{~d}, 1 \mathrm{H}, \mathrm{H}-6, J_{5,6}=8 \mathrm{~Hz}\right.$ ), $8.11(\mathrm{~d}, 2 \mathrm{H}, t$-butylbenzoyl, $J=9 \mathrm{~Hz}$ ). Anal. calcd for $\mathrm{C}_{42} \mathrm{H}_{44} \mathrm{O}_{8} \mathrm{~N}_{2}$ : C, 71.57; H, 6.29; N, 3.98. Found: C, 71.52; $\mathrm{H}, 6.33 ; \mathrm{N}, 4.01$.

3.1.5. $5^{\prime}$ - $O$-Dimethoxytrityl-(2' $\left.S\right)$-2' ${ }^{\prime}$-deoxy-2' $C$-methyl$N^{3}$-(4-t-butylbenzoyl)uridine (4b). Compound $3 \mathrm{~b}(2 \mathrm{~g}$, $5 \mathrm{mmol}$ ) was dimethoxytritylated and worked up as described for the synthesis of compound $\mathbf{4 a}$ above. Pure compound $\mathbf{4 b}$ was obtained as a foam $(73 \%) .{ }^{13} \mathrm{C}$ NMR spectrum of $\mathbf{4 b}\left(\mathrm{CDCl}_{3}\right)$ : $(\delta, \mathrm{ppm}) 169.3$ (t-butylbenzoyl); 161.3 (C-4); $158.5\left(\mathrm{C}_{\mathrm{Ar}}-\mathrm{OCH}_{3}\right.$, DMTr); 155.0 (t-butylbenzoyl); 149.1 (C-2); 143.9 (Ar, DMTr); 141.2 (C-6); 135.1, 129.8, 128.5, 128.0, 127.3 (Ar, DMTr+butylbenzoyl); 131.6, 124.4 ( $t$-butylbenzoyl); 113.2 (Ar, DMTr); 101.8 (C-5); 86.3 (C-Ar $\left.{ }_{3}, \mathrm{DMTr}\right) ; 86.1\left(\mathrm{C}-1^{\prime}\right)$; 83.7 (C-4'); $72.8 \quad\left(\mathrm{C}-3^{\prime}\right) ; 60.9\left(\mathrm{C}-5^{\prime}\right) ; 54.9\left(-\mathrm{OCH}_{3}\right.$, DMTr); 45.2 (C-2'); 34.6, 31.5 ( $t$-butylbenzoyl), $10.9\left(2^{\prime}-\right.$ methyl). ${ }^{1} \mathrm{H}$ NMR spectrum of $\mathbf{4 b}\left(\mathrm{CDCl}_{3}\right):(\delta, \mathrm{ppm}) 0.94$ $\left(\mathrm{d}, 3 \mathrm{H},-\mathrm{CH}_{3}, J_{\mathrm{CH} 3,2^{\prime}}=8 \mathrm{~Hz}\right) ; 1.32\left(\mathrm{~s}, 9 \mathrm{H},-\mathrm{CH}_{3} \mathrm{~s}, t\right.$-butylbenzoyl); 2.41 (brs, $\left.1 \mathrm{H}, \mathrm{OH}-3^{\prime}\right) ; 2.58\left(\mathrm{~m}, 1 \mathrm{H}, \mathrm{H}-2^{\prime}\right) ; 3.20$ $\left(\mathrm{dd}, 1 \mathrm{H}, \mathrm{H}-5^{\prime}, J_{5^{\prime}, 5^{\prime \prime}}=12 \mathrm{~Hz} ; J_{4^{\prime}, 5^{\prime \prime}}=1 \mathrm{~Hz}\right) ; 3.42(\mathrm{dd}, 1 \mathrm{H}, \mathrm{H}-$ $\left.5^{\prime \prime}, J_{5^{\prime}, 5^{\prime \prime}}=12 \mathrm{~Hz} ; J_{4^{\prime}, 5^{\prime \prime}}=1 \mathrm{~Hz}\right) ; 3.80,\left(\mathrm{~s}, 3 \mathrm{H},-\mathrm{OCH}_{3}\right) ; 3.82$, $\left(\mathrm{s}, 3 \mathrm{H},-\mathrm{OCH}_{3}\right) ; 4.05\left(\mathrm{~m}, 1 \mathrm{H}, \mathrm{H}-4^{\prime}\right) ; 4.11\left(\mathrm{~m}, 1 \mathrm{H}, \mathrm{H}-3^{\prime}\right)$; $5.32\left(\mathrm{~d}, 1 \mathrm{H}, \mathrm{H}-5, J_{5,6}=8 \mathrm{~Hz}\right) ; 6.28\left(\mathrm{~d}, 1 \mathrm{H}, \mathrm{H}-1^{\prime}, J_{1^{\prime}, 2^{\prime}}=8 \mathrm{~Hz}\right)$; $6.82\left(\mathrm{~d}, 4 \mathrm{H}, \mathrm{Ar}-\mathrm{OCH}_{3}\right.$, DMT, $\left.J=9 \mathrm{~Hz}\right) ; 7.20-7.35$ (m, 11 $\mathrm{H}, \mathrm{Ar}+\mathrm{Ar}-\mathrm{OCH}_{3}$, DMT and Ar, $t$-butylbenzoyl); 8.05 (d, $\left.1 \mathrm{H}, \mathrm{H}-6, J_{5,6}=8 \mathrm{~Hz}\right), 8.09$ (d, $2 \mathrm{H}, t$-butylbenzoyl, $J=9 \mathrm{~Hz}$ ). Anal. calcd for $\mathrm{C}_{42} \mathrm{H}_{44} \mathrm{O}_{8} \mathrm{~N}_{2}: \mathrm{C}, 71.57 ; \mathrm{H}, 6.29 ; \mathrm{N}, 3.98$. Found: C, 71.50; H, 6.25; N, 3.95.

3.1.6. $5^{\prime}$-O-Dimethoxytrityl-(2'R)-2'-deoxy-2'-C-methyl$N^{3}$-(4-t-butylbenzoyl)uridine-3'-O-(2-cyanoethyl $\quad N, N$ diisopropylphosphoramidite) (5a). $5^{\prime}$-O-Dimethoxytrityl$\left(2^{\prime} R\right)$-2'-deoxy-2'-C-methyl- $N^{3}$-(4-t-butylbenzoyl)uridine (4a) $(3.23 \mathrm{~g}, 5 \mathrm{mmol})$ was dried by evaporation of $\mathrm{CH}_{3} \mathrm{CN}$ and dissolved in dry 1,2-dichloroethane $(20 \mathrm{~mL})$ containing $\mathrm{N}, \mathrm{N}$-diisopropylethylamine $(10 \mathrm{mmol})$ under argon. 2Cyanoethoxy- $N, N$-diisopropylaminochlorophosphine $(6 \mathrm{mmol})$ was added drop wise with stirring. When TLC showed complete reaction, the mixture was quenched by addition of $\mathrm{MeOH}$. After $3 \mathrm{~min}$, dichloromethane $(50 \mathrm{~mL})$ was added and the solution was washed with $1 \mathrm{M}$ aqueous $\mathrm{Na}_{2} \mathrm{CO}_{3}(70 \mathrm{~mL})$ followed by saturated brine $(50 \mathrm{~mL})$. The organic phase was dried $\left(\mathrm{Na}_{2} \mathrm{SO}_{4}\right)$, filtered and evaporated to dryness. The residue was purified by silica gel column, eluting with $33 \%$ ethyl acetate in petrol containing $1 \%$ triethylamine to yield pure title compound $(78 \%) .{ }^{13} \mathrm{C}$ NMR spectrum of $\mathbf{5 a}\left(\mathrm{CDCl}_{3}\right)$ : $(\delta, \mathrm{ppm}) \quad 168.9 / 168.7$ ( $t$-butylbenzoyl); 161.9 (C-4); $158.7 / 158.6\left(\mathrm{C}_{\mathrm{Ar}}-\mathrm{OCH}_{3}\right.$, DMTr); 154.2 ( $t$-butylbenzoyl); 149.3 (C-2); 144.3/144.2 (Ar, DMTr); 140.2 (C-6); 135.3/135.2, 130.5/130.4/130.3, 128.6/128.4/128.2, 127.9/127.4/127.3 (Ar, DMTr+butylbenzoyl); 131.5, 124.4 ( $t$-butylbenzoyl); 117.4 (CN, phops);113.2/113.1 (Ar, DMTr); 102.1 (C-5); 87.0 $\left(\mathrm{C}-\mathrm{Ar}_{3}, \mathrm{DMTr}\right) ; 89.2 / 89.4\left(\mathrm{C}-1^{\prime}\right)$; $82.4\left(\mathrm{C}-4^{\prime}\right)$; 72.0/71.8 $\left(\mathrm{C}-3^{\prime}\right) ; 60.5\left(\mathrm{C}-5^{\prime}\right)$; 58.0/57.9 $\left(-\mathrm{OCH}_{2}\right.$, phosph); 55.2/55.1 ( $\left.-\mathrm{OCH}_{3}, \mathrm{DMTr}\right) ; 44.5 / 44.4$ (C-2'); 43.7/43.4 (CHs, phosp); 34.7, 31.3 (t-butylbenzoyl); $24.6\left(\mathrm{CH}_{3}\right.$, phosp); 18.9/18.8 $\left(\mathrm{CH}_{2}-\mathrm{CN}\right.$, phosp); 10.4/10.5 (2'-methyl). ${ }^{1} \mathrm{H}$ NMR spectrum of 5a $\left(\mathrm{CDCl}_{3}\right): \delta(\mathrm{ppm}) \quad 0.95 / 0.99\left(\mathrm{~d}, 3 \mathrm{H},-\mathrm{CH}_{3}\right.$, $\left.J_{\mathrm{CH} 3,2^{2}}=7 \mathrm{~Hz}\right) ; 1.08,1.15,1.16,1.17\left(\mathrm{~d}, 12 \mathrm{H},-\mathrm{CH}_{3}\right.$, phosp, $\left.J_{\mathrm{CH} 3, \mathrm{CH}}=7 \mathrm{~Hz}\right) ; 1.30 / 1.33\left(\mathrm{~s}, 9 \mathrm{H},-\mathrm{CH}_{3} \mathrm{~s}, t\right.$-butylbenzoyl); $2.28 / 2.30$, (m, 3H, $\mathrm{CH}_{2}-\mathrm{CN}$ and $\left.\mathrm{H}-2^{\prime}\right) ; 3.40-$ $3.45(\mathrm{~m}, 2 \mathrm{H},-\mathrm{CHs}$, phosp); 3.44-3.66 (m, 2H, H-5' and $\mathrm{H}-5^{\prime \prime} ; 3.85\left(\mathrm{~m}, 1 \mathrm{H}, \mathrm{H}-3^{\prime}\right) ; 3.79 / 3.80\left(\mathrm{~s}, 3 \mathrm{H},-\mathrm{OCH}_{3}\right)$; $3.81 / 3.82\left(\mathrm{~s}, 3 \mathrm{H},-\mathrm{OCH}_{3}\right) ; 3.83 / 3.86\left(\mathrm{~m}, 1 \mathrm{H},-\mathrm{CH}_{2} \mathrm{O}\right.$, phosp); 3.91/3.95 (m, $1 \mathrm{H},-\mathrm{CH}_{2} \mathrm{O}$, phosp); 4.40/4.42 (m, $\left.1 \mathrm{H}, \mathrm{H}-4^{\prime}\right) ; 5.20 / 5.25$ (d, $\left.1 \mathrm{H}, \mathrm{H}-5, J_{5,6}=8 \mathrm{~Hz}\right) ; 6.20 / 6.23$ (s, $\left.1 \mathrm{H}, \mathrm{H}-1^{\prime}\right)$; 6.81-6.88, 7.23-7.30, 7.35-7.45 (m, $17 \mathrm{H}$, $\mathrm{Ar}+\mathrm{Ar}-\mathrm{OCH}_{3}$, DMT and Ar, $t$-butylbenzoyl); 7.98/8.02 $\left(\mathrm{d}, \quad 1 \mathrm{H}, \quad \mathrm{H}-6, \quad \mathrm{~J}_{5,6}=8 \mathrm{~Hz}\right) .{ }^{31} \mathrm{P}$ NMR spectrum of $\mathbf{5 a}$ $\left(\mathrm{CDCl}_{3}\right):(\delta, \mathrm{ppm}) 147.20 ; 149.34$. Anal. calcd for $\mathrm{C}_{51} \mathrm{H}_{61} \mathrm{O}_{9} \mathrm{~N}_{4} \mathrm{P}: \mathrm{C}, 67.68 ; \mathrm{H}, 6.79 ; \mathrm{N}, 6.19$. Found: $\mathrm{C}$, $67.56 ; \mathrm{H}, 7.23 ; \mathrm{N}, 6.25$.

3.1.7. $5^{\prime}$-O $O$-Dimethoxytrityl-(2'S)-2'-deoxy-2' $C$-methyl$N^{3}$-(4-t-butylbenzoyl) uridine-3'-O-(2-cyanoethyl $N, N$ diisopropylphosphoramidite) (5b). Compound $4 \mathbf{b}$ (3.23 g, $5 \mathrm{mmol}$ ) was phosphitylated, worked up and purified according to the procedure used to prepare 5a above to 
yield the title compound $\mathbf{5 b}$ pure $(80 \%) .{ }^{13} \mathrm{C}$ NMR spectrum of $\mathbf{5 b}\left(\mathrm{CDCl}_{3}\right)$ : $(\delta, \mathrm{ppm})$ 169.0/168.9 ( $t$-butylbenzoyl); 161.5 (C-4); 158.7/158.6 ( $\left.\mathrm{C}_{\mathrm{Ar}}-\mathrm{OCH}_{3}, \mathrm{DMTr}\right) ; 154.9$ (t-butylbenzoyl); 149.2 (C-2); 144.4/144.2 (Ar, DMTr); 141.1 (C-6); 135.3/135.1, 130.5/130.3/130.2, 128.7/128.6/ 128.4, 127.7/127.4/127.3 (Ar, DMTr+butylbenzoyl); 131.4, 124.5 ( $t$-butylbenzoyl); 117.3 (CN, phops); 113.3/ 113.2 (Ar, DMTr); 102.0 (C-5); $86.5\left(\mathrm{C}-\mathrm{Ar}_{3}, \mathrm{DMTr}\right)$; 86.3/86.2 (C-1 $\left.{ }^{\prime}\right) ; 83.9 \quad\left(\mathrm{C}-4^{\prime}\right) ; 72.8 / 72.5 \quad\left(\mathrm{C}-3^{\prime}\right) ; 60.8$ $\left(\mathrm{C}-5^{\prime}\right) ; 57.9 / 57.6\left(-\mathrm{OCH}_{2}\right.$, phosph); 55.3/55.2 $\left(-\mathrm{OCH}_{3}\right.$, DMTr); 45.4/45.2 (C-2'); 43.8/43.5 (CHs, phosp); 34.6, 31.5 ( $t$-butylbenzoyl); $24.5\left(\mathrm{CH}_{3}\right.$, phosp); 18.9/18.6 $\left(\mathrm{CH}_{2}-\right.$ CN, phosp); 10.9/10.8 (2'-methyl). ${ }^{1} \mathrm{H}$ NMR spectrum of $\mathbf{5 b}$ $\left(\mathrm{CDCl}_{3}\right): \delta(\mathrm{ppm}) 0.99 / 1.02\left(\mathrm{~d}, 3 \mathrm{H},-\mathrm{CH}_{3}, J_{\mathrm{CH} 3,2^{\prime}}=7 \mathrm{~Hz}\right)$; $1.09,1.16,1.17,1.18\left(\mathrm{~d}, 12 \mathrm{H},-\mathrm{CH}_{3}\right.$, phosp, $\left.J_{\mathrm{CH} 3, \mathrm{CH}}=7 \mathrm{~Hz}\right)$; 1.31/1.32 (s, 9H, $-\mathrm{CH}_{3} \mathrm{~s}, t$-butylbenzoyl); 2.29/2.31, (m, $2 \mathrm{H}$, $\left.\mathrm{CH}_{2}-\mathrm{CN}\right) 2.55 / 2.60\left(\mathrm{~m}, 1 \mathrm{H}, \mathrm{H}-2^{\prime}\right) ; 3.39-3.44(\mathrm{~m}, 2 \mathrm{H}$, -CHs, phosp); 3.45-3.65 (m, 2H, H-5'and H-5-); 3.75 $\left(\mathrm{m}, 1 \mathrm{H}, \mathrm{H}-3^{\prime}\right) ; 3.80\left(\mathrm{~s}, 3 \mathrm{H},-\mathrm{OCH}_{3}\right) ; 3.81 / 3.83(\mathrm{~s}, 3 \mathrm{H}$, $\left.-\mathrm{OCH}_{3}\right) ; 3.83 / 3.85\left(\mathrm{~m}, 1 \mathrm{H}, \mathrm{CH}_{2} \mathrm{O}\right.$, phosp); 3.90/3.92 (m, $1 \mathrm{H},-\mathrm{CH}_{2} \mathrm{O}$, phosp); 4.35/4.40 (m, $\left.1 \mathrm{H}, \mathrm{H}-4^{\prime}\right) ; 5.12 / 5.15$ $\left(\mathrm{d}, 1 \mathrm{H}, \mathrm{H}-5, J_{5,6}=8 \mathrm{~Hz}\right) ; 6.25 / 6.29\left(\mathrm{~s}, 1 \mathrm{H}, \mathrm{H}-1^{\prime}\right) ; 6.80-$ $6.85,7.25-7.30,7.37-7.45\left(\mathrm{~m}, \quad 17 \mathrm{H}, \mathrm{Ar}+\mathrm{Ar}-\mathrm{OCH}_{3}\right.$, DMT and Ar, $t$-butylbenzoyl); 8.05/8.09 (d, 1H, H-6, $\left.J_{5,6}=8 \mathrm{~Hz}\right) .{ }^{31} \mathrm{P}$ NMR spectrum of $\mathbf{5 b}\left(\mathrm{CDCl}_{3}\right):(\delta, \mathrm{ppm})$ 145.94; 148.55. Anal. calcd for $\mathrm{C}_{51} \mathrm{H}_{61} \mathrm{O}_{9} \mathrm{~N}_{4} \mathrm{P}$ : C, 67.68; H, 6.79; N, 6.19. Found: C, 67.60; H, 7.83; N, 6.23.

3.1.8. $3^{\prime}, 5^{\prime}-O$-(Tetraisopropyldisiloxane-1,3-diyl)-(2'S)$2^{\prime}$-deoxy-2' $\boldsymbol{C}$-methyl-4- $\boldsymbol{O}$-(2-nitrophenyl) uridine (6). A mixture of $3^{\prime}, 5^{\prime}-O$-(tetraisopropyldisiloxane-1,3-diyl)$\left(2^{\prime} R\right)$ - and $-\left(2^{\prime} S\right)-2^{\prime}$-deoxy- $2^{\prime}-C$-methyluridine (1a and 1b) $(\sim 1: 3$, as determined by NMR, $10.1 \mathrm{~g}, 20.8 \mathrm{mmol})$ in $1,2-$ dichloroethane $(50 \mathrm{~mL})$ was treated with mesitylenesulfonyl chloride $(6.2 \mathrm{~g}, \quad 28.5 \mathrm{mmol})$, dimethylaminopyridine (930 mg, $7.6 \mathrm{mmol})$ and triethylamine $(13.4 \mathrm{~mL}, 96 \mathrm{mmol})$. After stirring for $1 \mathrm{~h}$, diazabicyclo [2.2.2] octane (DABCO) (424 mg, $2.8 \mathrm{mmol}$ ), 2-nitrophenol (7.92 g, $47 \mathrm{mmol}$ ) and triethylamine $(13.4 \mathrm{~mL}, 96 \mathrm{mmol})$ were added and stirring was continued for three more hours. The resulting solution was diluted with dichloromethane $(100 \mathrm{~mL})$ and poured in $1 \mathrm{M}$ aqueous $\mathrm{NaHCO}_{3}(500 \mathrm{~mL})$ with vigorous stirring. The organic layer was washed with $\mathrm{H}_{2} \mathrm{O}(2 \times 150 \mathrm{~mL})$, dried $\left(\mathrm{Na}_{2} \mathrm{SO}_{4}\right)$, filtered and evaporated in vacuo leaving a yellow foam that was purified by column chromatography on silica gel eluting with petroleum ether/ethyl acetate/dichloromethane $(10: 1: 10, \mathrm{v} / \mathrm{v} / \mathrm{v})$ to afford the $2^{\prime} S$-isomer $6(5.17 \mathrm{~g}$, $8.5 \mathrm{mmol})$ and the $2^{\prime} R$-isomer $(1.3 \mathrm{~g}, 2.1 \mathrm{mmol}) .{ }^{13} \mathrm{C} \mathrm{NMR}$ spectrum of $6\left(\mathrm{CDCl}_{3}\right)$ : $(\delta, \mathrm{ppm}) 170.2(\mathrm{C}-4) ; 154.8(\mathrm{C}-2)$; 145.0 (C-6); 144.7, 141.3, 134.6, 126.3, 125.6, 125.2 (2-nitro-

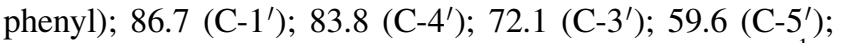
$43.9\left(\mathrm{C}-2^{\prime}\right)$; 17.1-12.2 (isopropyls), 11.0 (2'-methyl). ${ }^{1} \mathrm{H}$ NMR spectrum of $6\left(\mathrm{CDCl}_{3}\right):(\delta, \mathrm{ppm}) 0.80-1.10(\mathrm{~m}, 28 \mathrm{H}$, $-\mathrm{CHs}$ and $-\mathrm{CH}_{3} \mathrm{~s}$, disiloxane); $0.95\left(\mathrm{~d}, 3 \mathrm{H},-\mathrm{CH}_{3}\right.$, $\left.J_{\mathrm{CH} 3,2^{\prime}}=7 \mathrm{~Hz}\right) ; 2.70\left(\mathrm{~m}, 1 \mathrm{H}, \mathrm{H}-2^{\prime}\right) ; 3.80-4.25$ (m, 4H, H-3', $\mathrm{H}-4^{\prime}, \mathrm{H}-5^{\prime}$ and $\left.\mathrm{H}-5^{\prime \prime}\right) ; 6.19\left(\mathrm{~d}, 1 \mathrm{H}, \mathrm{H}-5, J_{5,6}=8 \mathrm{~Hz}\right) ; 6.32(\mathrm{~d}$, $\left.1 \mathrm{H}, \mathrm{H}-1^{\prime}, J_{1^{\prime}, 2^{\prime}}=6 \mathrm{~Hz}\right) ; 7.27$ (dd, $1 \mathrm{H}, \mathrm{H}-6$, nitrophenyl, $J_{6,5}=9 \mathrm{~Hz}, J_{6,4}=1 \mathrm{~Hz}$ ); 7.4 (ddd, 1H, H-4, nitrophenyl, $J_{4,5}=9 \mathrm{~Hz}, J_{4,3}=9 \mathrm{~Hz}, J_{6,4}=1 \mathrm{~Hz}$ ); 7.65 (ddd, $1 \mathrm{H}, \mathrm{H}-5$, nitrophenyl, $\left.J_{4,5}=9 \mathrm{~Hz}, J_{5,6}=9 \mathrm{~Hz}, J_{5.3}=1 \mathrm{~Hz}\right) ; 8.13(\mathrm{dd}, 1 \mathrm{H}, \mathrm{H}-3$, nitrophenyl, $\left.J_{4,3}=9 \mathrm{~Hz}, J_{5,3}=1 \mathrm{~Hz}\right) ; 8.25$ (d, $1 \mathrm{H}, \mathrm{H}-6$, $J_{5,6}=8 \mathrm{~Hz}$ ). Anal. calcd for $\mathrm{C}_{28} \mathrm{H}_{43} \mathrm{O}_{8} \mathrm{~N}_{3} \mathrm{Si}_{2}: \mathrm{C}$, 55.51; $\mathrm{H}$, 7.15; N, 6.94. Found: C, 55.63; H, 7.25; N, 6.84.
3.1.9. $\quad 3^{\prime}, 5^{\prime}-O$-(Tetraisopropyldisiloxane-1,3-diyl)-(2'S)$\mathbf{2}^{\prime}$-deoxy-2' $\boldsymbol{C}$-methyluridine (7). A mixture of 2-nitrobenzaldoxime $(880 \mathrm{mg}, 5.31 \mathrm{mmol})$ and tetramethylguanidine $(420 \mathrm{ml}, 3.32 \mathrm{mmol})$ in dry $\mathrm{CH}_{3} \mathrm{CN}(10 \mathrm{~mL})$ was poured over the solid compound 6 (2.02 g, $3.32 \mathrm{mmol})$ under anhydrous conditions and stirred at room temperature for $4 \mathrm{~h}$. The solvent was removed in vacuo to produce a syrup that was redissolved in dichloromethane $(200 \mathrm{~mL})$ and washed with $\mathrm{H}_{2} \mathrm{O}(3 \times 200 \mathrm{~mL})$. The organic layer was dried $\left(\mathrm{Na}_{2} \mathrm{SO}_{4}\right)$, filtered and evaporated in vacuo to give an oil which was purified by column chromatography on silica gel using dichloromethane/ethyl acetate (10:1 v/v) as eluent. The title compound was obtained in $93 \%$ yield $(1.50 \mathrm{~g})$. The spectroscopic data of this compound were in agreement with those previously reported. ${ }^{18}$. Anal. calcd for $\mathrm{C}_{22} \mathrm{H}_{40} \mathrm{O}_{6} \mathrm{~N}_{2} \mathrm{Si}_{2}$ : C, 54.51; H, 8.32; N, 5.78. Found: C, 54.61; H, 8.35; N, 5.84.

3.1.10. $5^{\prime}$ - $O$-Dimethoxytrityl- $\left(2^{\prime} S\right)-2^{\prime}$-Deoxy-2 $2^{\prime}-C$-methyluridine (8). $3^{\prime}, 5^{\prime}-O$-(Tetraisopropyldisiloxane-1,3-diyl)$\left(2^{\prime} S\right)-2^{\prime}$-deoxy-2'-C-methyluridine (7) $(0.95 \mathrm{~g}, 1.8 \mathrm{mmol})$ was desylilated as described above for the synthesis of compound 3a. The crude product was dimethoxytritylated, worked up and purified as described for the synthesis of compound $\mathbf{4 a}$ above. Pure compound $\mathbf{8}$ was obtained as a foam $(96 \%) .{ }^{13} \mathrm{C}$ NMR spectrum of $8\left(\mathrm{CDCl}_{3}\right):(\delta, \mathrm{ppm})$ 163.0 (C-4); $158.4\left(\mathrm{C}_{\mathrm{Ar}}-\mathrm{OCH}_{3}, \mathrm{DMTr}\right) ; 150.2$ (C-2); 144.2 (Ar, DMTr); 141.2 (C-6); 135.2, 129.6, 128.5, 128.0, 127.4 (Ar, DMTr); 113.0 (Ar, DMTr); 101.4 (C-5); $86.4\left(\mathrm{C}-\mathrm{Ar}_{3}, \mathrm{DMTr}\right) ; 86.1\left(\mathrm{C}-1^{\prime}\right) ; 82.9\left(\mathrm{C}-4^{\prime}\right) ; 72.7\left(\mathrm{C}-3^{\prime}\right)$;

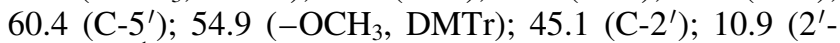
methyl). ${ }^{1} \mathrm{H}$ NMR spectrum of $8\left(\mathrm{CDCl}_{3}\right):(\delta, \mathrm{ppm}) 1.00$ $\left(\mathrm{d}, 3 \mathrm{H},-\mathrm{CH}_{3}, J_{\mathrm{CH} 3,2^{\prime}}=7 \mathrm{~Hz}\right.$ ); 2.40 (brs, $\left.1 \mathrm{H}, \mathrm{OH}-3^{\prime}\right) ; 2.55$ $\left(\mathrm{m}, 1 \mathrm{H}, \mathrm{H}-2^{\prime}\right) ; 3.18\left(\mathrm{dd}, 1 \mathrm{H}, \mathrm{H}-5^{\prime}, J_{5^{\prime}, 5^{\prime \prime}}=12 \mathrm{~Hz}, J_{5^{\prime \prime}, 4^{\prime}}=\right.$ $2 \mathrm{~Hz}) ; 3.50$ (dd, $\left.1 \mathrm{H}, \mathrm{H}-5^{\prime \prime}, J_{5^{\prime}, 5^{\prime \prime}}=12 \mathrm{~Hz}, J_{5^{\prime}, 4^{\prime}}=2 \mathrm{~Hz}\right) ; 3.83$ $\left(\mathrm{s}, 6 \mathrm{H},-\mathrm{OCH}_{3}\right) ; 4.00\left(\mathrm{~m}, 1 \mathrm{H}, \mathrm{H}-4^{\prime}\right) ; 4.12\left(\mathrm{dd}, 1 \mathrm{H}, \mathrm{H}-3^{\prime}\right.$, $\left.J_{3^{\prime}, 2^{\prime}}=J_{3^{\prime}, 4^{\prime}}=6 \mathrm{~Hz}\right) ; 5.30\left(\mathrm{~d}, 1 \mathrm{H}, \mathrm{H}-5, J_{5,6}=8 \mathrm{~Hz}\right) ; 6.29(\mathrm{~d}$, $\left.1 \mathrm{H}, \mathrm{H}-1^{\prime}, J_{1^{\prime}, 2^{\prime}}=8 \mathrm{~Hz}\right) ; 6.82\left(\mathrm{~d}, 4 \mathrm{H}, \mathrm{Ar}-\mathrm{OCH}_{3}, \mathrm{DMT}\right.$, $J=9 \mathrm{~Hz}) ; 7.20-7.35\left(\mathrm{~m}, 9 \mathrm{H}, \mathrm{Ar}+\mathrm{Ar}-\mathrm{OCH}_{3}, \mathrm{DMT}\right) ; 8.05$ (d, $1 \mathrm{H}, \mathrm{H}-6, J_{5,6}=8 \mathrm{~Hz}$ ); 9.35 (brs, $1 \mathrm{H}, \mathrm{NH}$ ). Anal. calcd for $\mathrm{C}_{31} \mathrm{H}_{32} \mathrm{O}_{7} \mathrm{~N}_{2}$ : C, 68.37; $\mathrm{H}, 5.92 ; \mathrm{N}$, 5.14. Found: $\mathrm{C}$, $68.45 ; \mathrm{H}, 6.00 ; \mathrm{N}, 5.23$.

3.1.11. $5^{\prime}-O$-Dimethoxytrityl- $\left(2^{\prime} S\right)-2^{\prime}$-deoxy-2 $2^{\prime}-C$-methyluridine- $3^{\prime}-O$-(2-cyanoethyl- $N, N$-diisopropylphosphoramidite) (9). Compound 8 (2.26, $5 \mathrm{mmol})$ was phosphitylated, worked up and purified according to the procedure used to prepare $\mathbf{5 a}$ above to yield the title compound 9 pure $(73 \%) .{ }^{13} \mathrm{C}$ NMR spectrum of 9 $\left(\mathrm{CDCl}_{3}\right):(\delta, \mathrm{ppm}) 162.9(\mathrm{C}-4) ; 158.8 / 158.7\left(\mathrm{C}_{\mathrm{Ar}}-\mathrm{OCH}_{3}\right.$, DMTr); 150.3 (C-2); 144.3/144.2 (Ar, DMTr); 141.1 (C-6); 135.3/135.1, 130.5/130.4/130.3, 128.9/128.6/128.4/ 128.2, 127.9/127.4/127.3 (Ar, DMTr); 117.4/117.3 (CN, phops);113.2 (Ar, DMTr); 101.8 (C-5); $87.0\left(\mathrm{C}-\mathrm{Ar}_{3}\right.$, DMTr); 86.4/86.2 (C-1'); $83.3\left(\mathrm{C}-4^{\prime}\right)$; 76.2/76.0 (C-3');

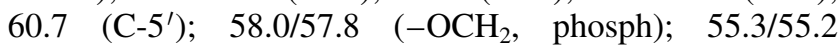
$\left(-\mathrm{OCH}_{3}, \mathrm{DMTr}\right) ; 44.8 / 44.7\left(\mathrm{C}-2^{\prime}\right) ; 43.9 / 43.3 / 43.2(\mathrm{CHs}$, phosp); 24.6/24.5 $\left(\mathrm{CH}_{3}\right.$, phosp); 17.4/17.3 $\left(\mathrm{CH}_{2}-\mathrm{CN}\right.$, phosp); 11.6/11.4 (2'-methyl). ${ }^{1} \mathrm{H}$ NMR spectrum of 9 $\left(\mathrm{CDCl}_{3}\right): \delta(\mathrm{ppm}) 1.00 / 1.05\left(\mathrm{~d}, 3 \mathrm{H},-\mathrm{CH}_{3}, J_{\mathrm{CH} 3,2^{\prime}}=7 \mathrm{~Hz}\right)$; $1.06,1.13,1.17,1.18\left(\mathrm{~d}, 12 \mathrm{H},-\mathrm{CH}_{3}\right.$, phosp, $\left.J_{\mathrm{CH} 3 \mathrm{CH}}=7 \mathrm{~Hz}\right)$; 2.28/2.29 (m, 2H, $\left.\mathrm{CH}_{2}-\mathrm{CN}\right) ; 2.60 / 2.63\left(\mathrm{~m}, 1 \mathrm{H}, \mathrm{H}-2^{\prime}\right)$; 3.39-3.46 (m, 2H, -CHs, phosp); 3.48-3.61 (m, 2H, H-5' 
and $\left.\mathrm{H}-5^{\prime \prime}\right) ; 3.70\left(\mathrm{~m}, 1 \mathrm{H}, \mathrm{H}-4^{\prime}\right) ; 3.81\left(\mathrm{~s}, 3 \mathrm{H},-\mathrm{OCH}_{3}\right) ; 3.80 /$ $3.79\left(\mathrm{~s}, 3 \mathrm{H},-\mathrm{OCH}_{3}\right) ; 3.80 / 3.85\left(\mathrm{~m}, 1 \mathrm{H}, \mathrm{CH}_{2} \mathrm{O}\right.$, phosp); $3.91 / 3.96\left(\mathrm{~m}, 1 \mathrm{H}, \mathrm{CH}_{2} \mathrm{CO}\right.$, phosp); 4.33/4.41 (m, 1H, H$\left.3^{\prime}\right) ; 5.10 / 5.16\left(\mathrm{~d}, 1 \mathrm{H}, \mathrm{H}-5, J_{5,6}=8 \mathrm{~Hz}\right) ; 6.29 / 6.30(\mathrm{~s}, 1 \mathrm{H}$, $\mathrm{H}-1^{\prime}$ ); 6.82-6.87, 7.22-7.30, 7.35-7.42 (m, 13H, DMT); 8.03/8.07 (d, 1H, H-6, $\left.J_{5,6}=8 \mathrm{~Hz}\right) ; 8.50$ (brs, $\left.1 \mathrm{H}, \mathrm{NH}\right) .{ }^{31} \mathrm{P}$ NMR spectrum of $9\left(\mathrm{CDCl}_{3}\right)$ : $(\delta$, ppm) $145.87 ; 148.51$. Anal. calcd for $\mathrm{C}_{40} \mathrm{H}_{49} \mathrm{O}_{8} \mathrm{~N}_{4} \mathrm{P}$ : C, 64.50; H, 6.63; N, 7.52. Found: C, 64.55; H, 6.57; N, 7.58.

3.1.12. $3^{\prime}, 5^{\prime}-O$-(Tetraisopropyldisiloxane-1,3-diyl)-(2'S)$2^{\prime}$-deoxy-2' $C$-methyl- $N^{4}$-isobutyryl cytidine (10). $3^{\prime}, 5^{\prime}-$ $O$-(Tetraisopropyldisiloxane-1,3-diyl)-(2'S)-2'-deoxy- $2^{\prime}-C$ methyl-4-O-(2-nitrophenyl) uridine (6) $(2,04 \mathrm{~g}, 3.36 \mathrm{mmol})$ in dry tetrahydrofuran $(120 \mathrm{~mL})$ was treated at room temperature with ammonia (10 bar) in a stainless steel bomb for four days. The solvent was evaporated in vacuo, the residue was dissolved in dry pyridine $(30 \mathrm{~mL})$ and the solution cooled in an ice bath. Isobutyrylchloride $(1.05 \mathrm{~mL}$, $10.12 \mathrm{mmol}$ ) was added with stirring and exclusion of moisture and after two hours the reaction was completed. The reaction mixture was evaporated to dryness, redissolved in dichloromethane $(100 \mathrm{~mL})$ and washed with $10 \%$ aqueous $\mathrm{NaHCO}_{3}(2 \times 100 \mathrm{~mL})$ and $\mathrm{H}_{2} \mathrm{O}(2 \times 100 \mathrm{~mL})$. The organic phase was dried $\left(\mathrm{Na}_{2} \mathrm{SO}_{4}\right)$, filtered and evaporated to dryness. The residue was purified by column chromatography using dichloromethane/MeOH (99:1, v/v) as eluant to afford pure compound $\mathbf{1 0}$ in $88 \%$ yield $(1.62 \mathrm{~g}) .{ }^{13} \mathrm{C} \mathrm{NMR}$ spectrum of $\mathbf{1 0}\left(\mathrm{CDCl}_{3}\right)$ : $(\delta, \mathrm{ppm}) 177.5$ (CO isobutyryl); 162.7 (C-4); 155.4 (C-2); 144.9 (C-6); 96.4 (C-5); 86.7 $\left(\mathrm{C}-1^{\prime}\right) ; 84.1\left(\mathrm{C}-4^{\prime}\right) ; 72.3\left(\mathrm{C}-3^{\prime}\right) ; 59.7\left(\mathrm{C}-5^{\prime}\right) ; 44.1\left(\mathrm{C}-2^{\prime}\right)$; $36.1,19.0$ (isobutyryl); $17.3-12.5$ (isopropyls), $11.3\left(2^{\prime}-\right.$ methyl). ${ }^{1} \mathrm{H}$ NMR spectrum of $\mathbf{1 0}\left(\mathrm{CDCl}_{3}\right):(\delta, \mathrm{ppm})$ $0.80-1.20\left(\mathrm{~m}, 28 \mathrm{H},-\mathrm{CHs}\right.$ and $-\mathrm{CH}_{3} \mathrm{~s}$, disiloxane); 0.95 $\left(\mathrm{d}, 3 \mathrm{H},-\mathrm{CH}_{3}, J_{\mathrm{CH} 3,2^{\prime}}=7 \mathrm{~Hz}\right) ; 1.28\left(\mathrm{~d}, 6 \mathrm{H},-\mathrm{CH}_{3}\right.$, isobutyryl, $\left.J_{\mathrm{CH}, \mathrm{CH} 3}=7 \mathrm{~Hz}\right) ; 2.50\left(\mathrm{~m}, 1 \mathrm{H}, \mathrm{H}-2^{\prime}\right) ; 2.65(\mathrm{q}, 1 \mathrm{H},-\mathrm{CH}$, isobutyryl, $\left.J_{\mathrm{CH}, \mathrm{CH} 3}=7 \mathrm{~Hz}\right) ; 3.65-4.20\left(\mathrm{~m}, 3 \mathrm{H}, \mathrm{H}-4^{\prime}, \mathrm{H}-5^{\prime}\right.$ and $\left.\mathrm{H}-5^{\prime \prime}\right) ; 4.25\left(\mathrm{~m}, 1 \mathrm{H}, \mathrm{H}-3^{\prime}\right) ; 6.18\left(\mathrm{~s}, 1 \mathrm{H}, \mathrm{H}-1^{\prime}\right) ; 6.95(\mathrm{~d}$, $1 \mathrm{H}, \mathrm{H}-5, J_{5,6}=8 \mathrm{~Hz}$ ); 8.00 (d, $1 \mathrm{H}, \mathrm{H}-6, J_{5,6}=8 \mathrm{~Hz}$ ); 9.58 (brs, $1 \mathrm{H}, \mathrm{NH})$. Anal. calcd for $\mathrm{C}_{26} \mathrm{H}_{47} \mathrm{O}_{6} \mathrm{~N}_{3} \mathrm{Si}_{2}: \mathrm{C}, 56.39 ; \mathrm{H}, 8.55$; N, 7.59. Found: C, 56.44; H, 8.51; N, 7.49.

3.1.13. 5' - $O$-Dimethoxytrityl- $\left(2^{\prime} S\right)-2^{\prime}$-Deoxy-2' $C$-methyl$N^{4}$-isobutyrylcytidine (11). $3^{\prime}, 5^{\prime}-O$-(Tetraisopropyldisiloxane-1,3-diyl)-(2'S)-2'-deoxy-2'-C-methyl- $N^{4}$-isobutyryl cytidine (10) was desilylated and worked up according to procedures used to prepare $\mathbf{3} \mathbf{a}$ and the crude product were dimethoxytritylated according to procedures given for the synthesis of 4a. The residue was purified by column chromatography which was first washed with $50 \%$ ethyl acetate in petrol and then pure compound $\mathbf{1 1}$ was eluted by using $2 \% \mathrm{MeOH}$ in dichloromethane (79\%).

${ }^{13} \mathrm{C}$ NMR spectrum of $\mathbf{1 1}\left(\mathrm{CDCl}_{3}\right):(\delta, \mathrm{ppm}) 177.1(\mathrm{CO}$ isobutyryl); $162.3(\mathrm{C}-4) ; 158.5\left(\mathrm{C}_{\mathrm{Ar}}-\mathrm{OCH}_{3}, \mathrm{DMTr}\right) ; 155.6$ (C-2); 143.2 (Ar, DMTr); 145.7 (C-6); 135.1, 129.7, 128., 128.0, 127.4 (Ar, DMTr); 113.0 (Ar, DMTr); 96.4 (C-5); $87.1\left(\mathrm{C}-1^{\prime}\right) ; 86.4\left(\mathrm{C}-\mathrm{Ar}_{3}, \mathrm{DMTr}\right) ; 83.6\left(\mathrm{C}-4^{\prime}\right) ; 73.7\left(\mathrm{C}-3^{\prime}\right)$; $61.3\left(\mathrm{C}-5^{\prime}\right) ; 54.9\left(-\mathrm{OCH}_{3}, \mathrm{DMTr}\right) ; 45.9\left(\mathrm{C}-2^{\prime}\right) ; 35.9,18.8$ (isobutyryl); 10.9 (2'-methyl). ${ }^{1} \mathrm{H}$ NMR spectrum of $\mathbf{1 1}$ $\left(\mathrm{CDCl}_{3}\right)$ : $(\delta, \mathrm{ppm}) 0.94\left(\mathrm{~d}, 3 \mathrm{H},-\mathrm{CH}_{3}, J_{\mathrm{CH} 3,2^{\prime}}=7 \mathrm{~Hz}\right) ; 1.25$ $\left(\mathrm{d}, 6 \mathrm{H},-\mathrm{CH}_{3}\right.$, isobutyryl, $\left.J_{\mathrm{CH}, \mathrm{CH} 3}=7 \mathrm{~Hz}\right) ; 2.29(\mathrm{~m}, 1 \mathrm{H}$, $\left.\mathrm{H}-2^{\prime}\right) ; 2.65\left(\mathrm{q}, 1 \mathrm{H},-\mathrm{CH}\right.$, isobutyryl, $\left.J_{\mathrm{CH}, \mathrm{CH} 3}=7 \mathrm{~Hz}\right) ; 2.67$ (brs, $\left.1 \mathrm{H}, \mathrm{OH}-3^{\prime}\right) ; 3.54\left(\mathrm{~m}, 2 \mathrm{H}, \mathrm{H}-5^{\prime}\right.$ and $\left.\mathrm{H}-5^{\prime \prime}\right) ; 3.75$ (s, 6H, $\left.-\mathrm{OCH}_{3}\right) ; 3.81\left(\mathrm{~m}, 1 \mathrm{H}, \mathrm{H}-4^{\prime}\right) ; 4.27\left(\mathrm{~m}, 1 \mathrm{H}, \mathrm{H}-3^{\prime}\right) ; 6.21(\mathrm{~s}$, $\left.1 \mathrm{H}, \mathrm{H}-1^{\prime}\right) ; 6.84\left(\mathrm{~d}, 4 \mathrm{H}, \mathrm{Ar}-\mathrm{OCH}_{3}, \mathrm{DMT}, J=9 \mathrm{~Hz}\right) ; 6.98(\mathrm{~d}$, $\left.1 \mathrm{H}, \mathrm{H}-5, J_{5,6}=8 \mathrm{~Hz}\right) ; 7.18-7.40\left(\mathrm{~m}, 9 \mathrm{H}, \mathrm{Ar}+\mathrm{Ar}-\mathrm{OCH}_{3}\right.$, DMT); 8.02 (d, $\left.1 \mathrm{H}, \mathrm{H}-6, J_{5,6}=8 \mathrm{~Hz}\right) ; 8.30$ (brs, $1 \mathrm{H}, \mathrm{NH}$ ). Anal. calcd for $\mathrm{C}_{35} \mathrm{H}_{39} \mathrm{O}_{7} \mathrm{~N}_{3}$ : C, 64.50; H, 6.41; N, 6.85. Found: C, 64.57; H, 6.46; N, 6.89 .

3.1.14. 5' $-O$-Dimethoxytrityl-(2'S)-2'-deoxy-2'-C -methyl$N^{4}$-isobutyrylcytidine-3'-O-(2-cyanoethyl- $N, N$-diisopropylphosphoramidite) (12). Compound 11 (3.01 g, $5 \mathrm{mmol}$ ) was phosphitylated, worked up according to the procedure used to prepare 5a above. The crude product was purified by column chromatography on silica gel eluting with $30 \%$ ethyl acetate in dichloromethane containing $1 \%$ of triethylamine to yield the title compound 12 pure $(72 \%) .{ }^{13} \mathrm{C}$ NMR spectrum of $\mathbf{1 2}\left(\mathrm{CDCl}_{3}\right):(\delta, \mathrm{ppm}) 177.0$ (CO, isobutyryl); 162.1 (C-4); 158.8/158.7 $\left(\mathrm{C}_{\mathrm{Ar}}-\mathrm{OCH}_{3}\right.$, DMTr); 155.3 (C-2); 145.6 (Ar, DMTr); 144.0 (C-6); 135.5/135.4, 130.4/130.4/130.3/130.2, 128.6/128.5, 127.9/ 127.3/127.1 (Ar, DMTr); 117.3 (CN, phops);113.2 (Ar, DMTr); 95.9 (C-5); 87.6/87.3 (C-Ar 3 , DMTr); 86.9 $\left(\mathrm{C}-1^{\prime}\right)$; 83.3 (C-4'); 76.9 (C-3'); 61.3/61.1 (C-5'); 58.1/ $58.0\left(-\mathrm{OCH}_{2}\right.$, phosph); $55.2\left(-\mathrm{OCH}_{3}, \mathrm{DMTr}\right) ; 44.6 / 44.5$ (C-2'); 43.3/43.2/43.1 (CHs, phosp); 36.7 (CH, isobutyryl); 24.6/24.5/24.4 $\left(\mathrm{CH}_{3}\right.$, phosp); 20.3/20.0 $\left(\mathrm{CH}_{3}\right.$, isobutyryl); $19.0\left(\mathrm{CH}_{2}-\mathrm{CN}\right.$, phosp); 11.8/11.6 (2'-methyl). ${ }^{1} \mathrm{H}$ NMR spectrum of $12\left(\mathrm{CDCl}_{3}\right)$ : $(\delta, \mathrm{ppm}) \quad 0.93 / 0.94(\mathrm{~d}, 3 \mathrm{H}$, $\left.-\mathrm{CH}_{3}, J_{\mathrm{CH} 3,2^{\prime}}=7 \mathrm{~Hz}\right) ; 1.00,1.131 .16\left(\mathrm{~d}, 12 \mathrm{H},-\mathrm{CH}_{3}\right.$, phosp, $\left.J_{\mathrm{CH} 3, \mathrm{CH}}=7 \mathrm{~Hz}\right) ; 1.20\left(\mathrm{~m}, 6 \mathrm{H},-\mathrm{CH}_{3}\right.$, isobutyryl); 2.29/2.30 (m, $\left.2 \mathrm{H}, \mathrm{CH}_{2}-\mathrm{CN}\right) ; 2.58\left(\mathrm{~m}, 1 \mathrm{H}, \mathrm{H}-2^{\prime}\right) ; 2.79(\mathrm{~m}$, $1 \mathrm{H},-\mathrm{CH}$, isobutyryl); 3.41-3.47 (m, $2 \mathrm{H},-\mathrm{CHs}$, phosp); $3.48-3.58\left(\mathrm{~m}, 2 \mathrm{H}, \mathrm{H}-5^{\prime}\right.$ and $\left.\mathrm{H}-5^{\prime \prime}\right) ; 3.61 / 3.63(\mathrm{~m}, 1 \mathrm{H}$, $\left.\mathrm{H}-4^{\prime}\right) ; 3.80\left(\mathrm{~s}, 3 \mathrm{H},-\mathrm{OCH}_{3}\right) ; 3.82\left(\mathrm{~s}, 3 \mathrm{H},-\mathrm{OCH}_{3}\right) ; 3.75 /$ $3.87\left(\mathrm{~m}, 1 \mathrm{H}, \mathrm{CH}_{2} \mathrm{O}\right.$, phops $) ; 3.97 / 4.04\left(\mathrm{~m}, 1 \mathrm{H}, \mathrm{CH}_{2} \mathrm{O}\right.$, phosp); 4.21/4.30 (m, 1H, H-3'); 6.39/6.41 (d, 1H, H-1', $\left.J_{1^{\prime}-2^{\prime}}=7 \mathrm{~Hz}\right) ; 6.97 / 7.04\left(\mathrm{~d}, 1 \mathrm{H}, \mathrm{H}-5, J_{5,6}=7 \mathrm{~Hz}\right) ; 6.82$ 6.88, 7.25-7.35, 7.38-7.42 (m, 13H, DMT); 8.29 (brs, $1 \mathrm{H}, \mathrm{NH}) ; 8.34 / 8.40\left(\mathrm{~d}, 1 \mathrm{H}, \mathrm{H}-6, J_{5,6}=7 \mathrm{~Hz}\right)$.

${ }^{31} \mathrm{P}$ NMR spectrum of $\mathbf{1 2}\left(\mathrm{CDCl}_{3}\right)$ : $(\delta, \mathrm{ppm}) 145.88 ; 148.39$. Anal. calcd for $\mathrm{C}_{44} \mathrm{H}_{56} \mathrm{O}_{8} \mathrm{~N}_{5} \mathrm{P}: \mathrm{C}, 64.93 ; \mathrm{H}, 6.94 ; \mathrm{N}, 8.60$. Found: C, 64.98; H, 7.02; N, 8.66.

\section{Acknowledgements}

We thank Consejo Nacional de Investigaciones Científicas y Técnicas-Argentina, Universidad Nacional de QuilmesArgentina and International Centre for Genetic Ingeneering and Biotechnology-Trieste, Italy for financial support.

\section{References}

1. Varma, S.; Eckstein, F. Аnпu. Rev. Biochem. 1998, 67, 99134.

2. Capeans, C.; Pineiro, A.; Dominguez, F.; Loidi, L.; Buceta, M.; Carneiro, C.; Garcia-Caballero, T. Exp. Eye Res. 1998, 66, 581-589.

3. Shinozuka, K.; Okamoto, T.; Matsukura, M.; Sawai, H. Nucl. Acids Symp. Ser. 1997, 37, 215-216.

4. Stein, C. A.; Cheng, Y. C. Science 1993, 261, 1004-1012. 
5. Freier, S. M.; Altmann, K. H. Nucl. Acids Res. 1997, 25, 4429-4443.

6. Grøtli, M.; Douglas, M.; Eritja, R.; Sproat, B. S. Tetrahedron 1998, 54, 5899-5914.

7. Iribarren, A. M.; Sproat, B. S.; Neuner, P.; Sulston, I.; Ryder, U.; Lamond, A. I. Proc. Natl. Acad. Sci. USA 1990, 87, 77477751.

8. Shibara, S.; Mukai, S.; Morisawa, H.; Nakashima; Kobayashi, S.; Yamamoto, N. Nucl. Acids Res. 1989, 17, 239-252.

9. Cummins, L. L.; Owens, S. R.; Risen, L. M.; Lesnik, E. A.; Freier, S. M.; McGee, D.; Guinosso, C. J.; Dan Cook, P. Nucl. Acids Res. 1995, 23, 2019-2024.

10. Lesnik, E. A.; Guinosso, C. J.; Kawasaki, A. M.; Sasmor, H.; Zounes, M.; Cummins, L. L.; Ecker, D. J.; Dan Cook, P.; Freier, S. M. Biochemistry 1993, 32, 7832-7838.

11. Saenger, W. In Principles of Nucleic Acid Structure; Cantor, C. R., Ed.; Springer: New York, 1988; pp. 69-78.

12. Cicero, D. O.; Iribarren, A. M.; Bazzo, R. Appl. Magn. Reson. 1994, 7, 95-105.

13. Koole, L. H.; Wu, J.; Neidle, S.; Chattopadhyaya, J. J. Am. Chem. Soc. 1992, 114, 2687-2696.
14. Schmidt, C.; Bévierre, M. O.; De Mesmaeker, A.; Altmann, K. H. Bioorg. Med. Chem. Lett. 1994, 4, 1969-1974.

15. Beigelman, L.; Mc Swiggen, J. A.; Draper, K. G.; Gonzalez, C.; Jenser, K.; Karpeisky, A. M.; Modak, A. S.; MatulicAdamic, J.; Di Renzo, A. B.; Haeberli, P.; Sweedler, D.; Tracz, D.; Grimm, S.; Wincott, F. E.; Thackray, V. G.; Usman, N. J. Biol. Chem. 1995, 270, 25702-25708.

16. Beigelman, L.; Karpeisky, A. M.; Matulic-Adamic, J.; Haeberli, P.; Sweedler, D.; Usman, N. Nucl. Acids Res. 1995, 23, 4434-4442.

17. Dayringer, H. E.; Tramontano, A.; Spang, S. R.; Fletterick, R. J. J. Mol. Graphics 1986, 4, 82-87.

18. Cicero, D.; Neuner, P.; Franzese, O.; D'onofrio, C.; Iribarren, A. M. Bioorg. Med. Chem. Lett. 1994, 4, 861-866.

19. Brown, T.; Brown, D. J. S. In Oligonucleotides and Analogues-A Practical Approach; Eckstein, F., Ed.; IRL Press: Oxford, 1992; p 78.

20. Manoharan, M. Biochim. Biophys. Acta 1999, 1489, 117-130.

21. Sekine, M. J. Org. Chem. 1989, 54, 2321-2326. 